\title{
Reactivity and Selectivity of 1,3-Diyn-6-enes in Electrophilic Transition Metal-Catalyzed Reactions
}

\author{
Eun Jin Cho, Mansuk Kim, and Daesung Lee* \\ Department of Chemistry, University of Wisconsin, Madison, WI 53706
}

Supporting Information

$\begin{array}{ll}\text { General } & 2\end{array}$

Representative procedures for the synthesis of diynes 2

Characterization information for substrates 3

General procedure for $\mathrm{PtCl}_{2}$-catalyzed reactions $\quad 6$

$\begin{array}{ll}\text { Characterization information for products } & 6\end{array}$

${ }^{1} \mathrm{H}$ NMR and ${ }^{13} \mathrm{C}$ NMR spectra $\quad 12$ 


\section{Materials and Methods}

${ }^{1} \mathrm{H}$ and ${ }^{13} \mathrm{C}$ NMR spectra were recorded on a Varian Mercury plus 300 or a Bruker AC-300 spectrometer. Tetramethylsilane (TMS) served as an internal standard $(\delta=0)$ for ${ }^{1} \mathrm{H}$ NMR and $\mathrm{CDCl}_{3}$ was used as an internal standard $(\delta=77.23)$ for ${ }^{13} \mathrm{C}$ NMR. Flasks were ovendried overnight and cooled under a stream of nitrogen. Compounds were purchased from Aldrich or Strem. THF was distilled from sodium/benzophenone and dichloromethane was distilled from $\mathrm{CaH}_{2}$. Flash chromatography was performed using silica gel $60 \AA$ (32-63 mesh) from Sorbent Technologies. Reactions were monitored by thin layer chromatography (TLC) using $0.25 \mathrm{~mm}$ E. Merck pre-coated silica gel 60 (particle size 0.040-0.063 mm).

\section{Representative procedures for the synthesis of diynes ${ }^{1}$}

Alkyne-Bromoalkyne Coupling: Copper(I) chloride (0.2 equiv) was added to a stirred solution of $n-\mathrm{BuNH}_{2}(1.2 \mathrm{~mL})$ and distilled water $(2.8 \mathrm{~mL})$ at $0{ }^{\circ} \mathrm{C}$, which resulted in a deep blue solution. A few crystals of $\mathrm{NH}_{2} \mathrm{OH} \cdot \mathrm{HCl}$ were added to get a colorless solution which is indicative of the presence of the required $\mathrm{Cu}(\mathrm{I})$ salt. At the same temperature was added diethyl 2-allyl-2-(prop-2-ynyl)malonate $(318 \mathrm{mg}, 1.34 \mathrm{mmol}$ ) by a syringe as a solution in $\mathrm{CH}_{2} \mathrm{Cl}_{2}$ upon which was a yellow suspension formed. Then a freshly prepared 3bromo-1-phenylprop-2-yn-1-ol (1.1 equiv) was slowly added under a flow of $\mathrm{N}_{2}$ in order to prevent the solution from turning to green or blue $\left(\mathrm{NH}_{2} \mathrm{OH} \cdot \mathrm{HCl}\right.$ can be added as necessary throughout the reaction). The reaction mixture was allowed to warm up to room temperature. After stirring for 10 20 minutes, the brownish orange colored solution was extracted twice with $\mathrm{CH}_{2} \mathrm{Cl}_{2}$, dried over magnesium sulfate, and concentrated under vacuum. Purification by flash chromatography on silica gel (hexane/ethyl ether) afforded the product (452 $\mathrm{mg}, 92 \%$ ).

Acetylation: To a solution of the corresponding product alcohol (452 $\mathrm{mg}, 1.23 \mathrm{mmol}$ ) in $\mathrm{CH}_{2} \mathrm{Cl}_{2}$ was added acetic anhydride (1.2 equiv), $\mathrm{Et}_{3} \mathrm{~N}$ (2.0 equiv), and $N, N-$ dimethylaminopyridine ( 0.05 equiv). The reaction mixture was stirred for 2 hours and quenched with aqueous $\mathrm{NH}_{4} \mathrm{Cl}$. This solution was extracted twice with $\mathrm{CH}_{2} \mathrm{Cl}_{2}$, dried over magnesium sulfate, and concentrated under vacuum. Purification by flash chromatography on silica gel (hexanes/ethyl ether) afforded the product 13b (495 mg, 98\%).

\footnotetext{
${ }^{1}$ (a) Marino, J. P.; Nguyen, H. N. J. Org. Chem. 2002, 67, 6841. (b) Kim, S.; Kim, S.; Lee, T.; Ko,
} H.; Kim, D. Org. Lett. 2004, 6, 3601, and references cited therein. 


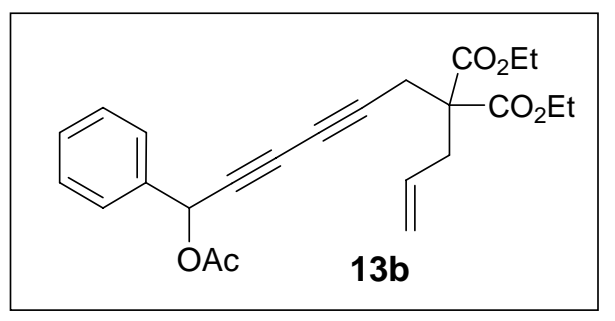

Colorless oil: ${ }^{1} \mathbf{H}$ NMR (300 $\left.\mathbf{M H z}, \mathbf{C D C l}_{3}\right) \delta$ 7.50-7.46 (m, 2H), 7.40-7.36 (m, 3H), $6.47(\mathrm{~s}, 1 \mathrm{H}), 5.61$ (ddt, $J=$ $17.1,10.0,7.4 \mathrm{~Hz}, 1 \mathrm{H}), 5.22-5.11(\mathrm{~m}, 2 \mathrm{H}), 4.20(\mathrm{q}, J=$ $7.2 \mathrm{~Hz}, 4 \mathrm{H}), 2.91(\mathrm{~s}, 2 \mathrm{H}), 2.79(\mathrm{~d}, J=7.4 \mathrm{~Hz}, 2 \mathrm{H}), 2.09$ (s, 3H), $1.25(\mathrm{t}, J=7.2 \mathrm{~Hz}, 6 \mathrm{H}) ;{ }^{13} \mathbf{C}$ NMR (75 MHz, $\mathbf{C D C l}_{3}$ ) $\delta 169.63,169.47(2), 136.39,131.63,129.27,128.88(2)$, 127.84(2), 120.25, 77.03, 72.60, 71.76, 67.25, 65.87, 61.96(2), 56.80, 36.81, 23.69, 21.05, 14.18(2); IR (neat): $v_{\max }=2983,2260,1733,1218 \mathrm{~cm}^{-1}$; HRMS $\boldsymbol{m} / \mathbf{z}$ (ESI): calc. for $\mathrm{C}_{24} \mathrm{H}_{26} \mathrm{O}_{6} \mathrm{Na}\left[\mathrm{M}^{+}+\mathrm{Na}\right] 433.1627$, found 433.1606 .

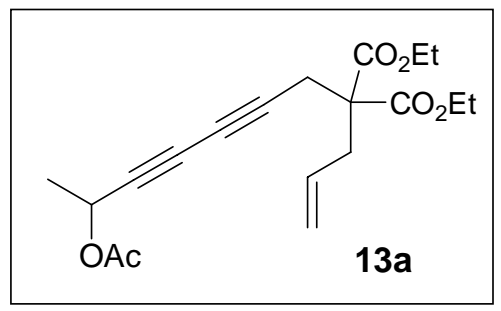

Colorless oil: ${ }^{1} \mathbf{H}$ NMR (300 MHz, $\mathbf{C D C l}_{3}$ ) $\delta 5.60$ (ddt, $J=16.8$, 9.8, 7.5Hz, 1H), 5.45 (q, $J=6.5 \mathrm{~Hz}, 1 \mathrm{H}), 5.23-5.12(\mathrm{~m}, 2 \mathrm{H})$, $4.21(\mathrm{q}, J=7.1 \mathrm{~Hz}, 4 \mathrm{H}), 2.89(\mathrm{~s}, 2 \mathrm{H}), 2.78(\mathrm{~d}, J=7.5 \mathrm{~Hz}, 2 \mathrm{H})$, 2.07 (s, 3H), 1.49 (d, $J=6.5 \mathrm{~Hz}, 3 \mathrm{H}), 1.26$ (t, $J=7.1 \mathrm{~Hz}, 6 \mathrm{H})$; ${ }^{13} \mathbf{C}$ NMR (75 MHz, $\left.\mathbf{C D C l}_{3}\right) \delta 169.86,169.58(2), 131.70$, $120.29,76.25,74.56,69.55,67.30,62.00(2), 60.57,56.86,36.84$, 23.69, 21.24, 21.11, 14.24(2); IR (neat): $v_{\max }=2985,2261$, 1736, $1224 \mathrm{~cm}^{-1}$; HRMS $\mathbf{m} / \mathbf{z}$ (ESI): calc. for $\mathrm{C}_{19} \mathrm{H}_{24} \mathrm{O}_{6} \mathrm{Na}\left[\mathrm{M}^{+}+\mathrm{Na}\right] 371.1471$, found 371.1462 .

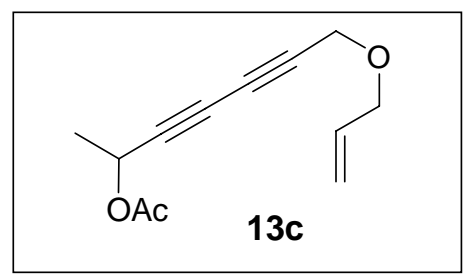

Colorless oil: ${ }^{1} \mathbf{H}$ NMR (300 MHz, $\mathbf{C D C l}_{3}$ ) $\delta 5.88$ (ddt, $J=17.2$, $10.3,5.8 \mathrm{~Hz}, 1 \mathrm{H}$ ), 5.48 (q, $J=6.8 \mathrm{~Hz}, 1 \mathrm{H}$ ), 5.31 (ddt, $J=17.2,1.6$, $1.6 \mathrm{~Hz}, 1 \mathrm{H}), 5.25-5.20(\mathrm{~m}, 1 \mathrm{H}), 4.22(\mathrm{~s}, 2 \mathrm{H}), 4.06(\mathrm{dt}, J=5.8,1.6$ $\mathrm{Hz}, 2 \mathrm{H}), 2.07$ (s, 3H), $1.51(\mathrm{~d}, J=6.8 \mathrm{~Hz}, 3 \mathrm{H}) ;{ }^{13} \mathrm{C}$ NMR (75 MHz, $\left.\mathbf{C D C l}_{3}\right) \delta 169.81,133.83,118.32,77.17,76.40,70.93,70.27$, 68.97, 60.47, 57.66, 21.12, 21.05; HRMS m/z (ESI): calc. for $\mathrm{C}_{12} \mathrm{H}_{14} \mathrm{O}_{3} \mathrm{Na}\left[\mathrm{M}^{+}+\mathrm{Na}\right] 229.0841$, found 229.0848.

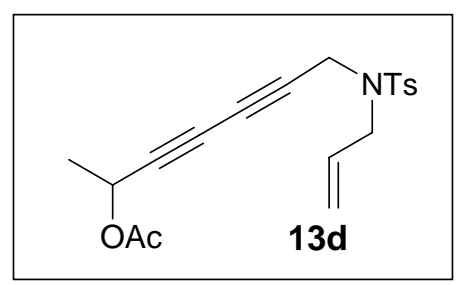

Colorless oil: ${ }^{1} \mathbf{H}$ NMR (300 MHz, $\left.\mathbf{C D C l}_{3}\right) \delta 7.72(\mathrm{~d}, J=8.2 \mathrm{~Hz}$, $2 \mathrm{H}), 7.33$ (d, $J=8.2 \mathrm{~Hz}, 2 \mathrm{H}), 5.71$ (ddt, $J=17.0,10.5,6.5 \mathrm{~Hz}, 1 \mathrm{H})$, 5.42 (q, $J=6.8 \mathrm{~Hz}, 1 \mathrm{H}), 5.31-5.23(\mathrm{~m}, 2 \mathrm{H}), 4.14$ (s, 2H), 3.78 (d, $J$ $=6.5 \mathrm{~Hz}, 2 \mathrm{H}), 2.44(\mathrm{~s}, 3 \mathrm{H}), 2.08(\mathrm{~s}, 3 \mathrm{H}), 1.47(\mathrm{~d}, J=6.8 \mathrm{~Hz}, 3 \mathrm{H})$; ${ }^{13}$ C NMR (75 MHz, $\left.\mathbf{C D C l}_{3}\right) \delta 169.74,143.98,135.69,131.88$, 129.81(2), 127.90(2), 120.46, 76.48, 73.22, 69.52, 68.62, 60.32, 49.61, 36.65, 21.70, 21.18, 21.06; IR (neat): $v_{\max }=2924,1744,1226,1164 \mathrm{~cm}^{-1} ; \mathbf{H R M S ~ m / z}$ (ESI): calc. for $\mathrm{C}_{19} \mathrm{H}_{21} \mathrm{NO}_{4} \mathrm{SNa}\left[\mathrm{M}^{+}+\mathrm{Na}\right] 382.1089$, found 382.1100 . 


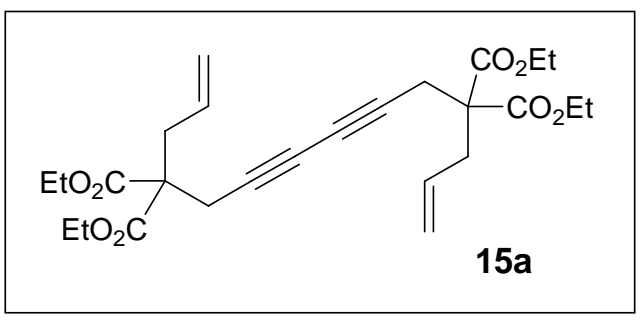

Colorless oil: ${ }^{\mathbf{1}} \mathbf{H}$ NMR (300 $\left.\mathbf{M H z}, \mathbf{C D C l}_{3}\right) \delta 5.61$ (ddt, $J=17.2,9.9,7.2 \mathrm{~Hz}, 2 \mathrm{H}), 5.21-5.11(\mathrm{~m}, 4 \mathrm{H}), 4.21$ (q, $J$ $=7.2 \mathrm{~Hz}, 8 \mathrm{H}), 2.86(\mathrm{~s}, 4 \mathrm{H}), 2.78(\mathrm{~d}, J=7.2 \mathrm{~Hz}, 4 \mathrm{H})$, $1.26(\mathrm{t}, J=7.2 \mathrm{~Hz}, 12 \mathrm{H}) ;{ }^{13} \mathrm{C}$ NMR (75 $\mathbf{M H z}, \mathbf{C D C l}_{3}$ ) $\delta$ 169.66(4), 131.81(2), 120.14(2), 72.67(2), 68.04(2), 61.92(4), 56.93(2), 36.85(2), 23.65(2), 14.22(4); IR (neat): $v_{\max }=2983,2261,1735,1215 \mathrm{~cm}^{-1}$; HRMS m/z (ESI): calc. for $\mathrm{C}_{26} \mathrm{H}_{34} \mathrm{O}_{8} \mathrm{Na}\left[\mathrm{M}^{+}+\mathrm{Na}\right]$ 497.2151, found 497.2153.

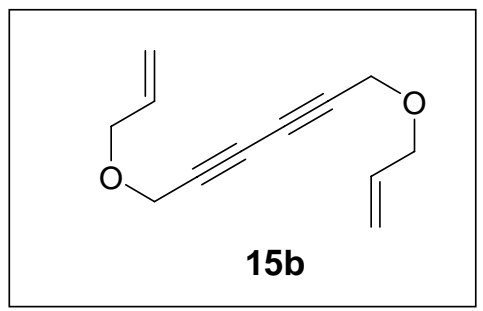

190.0994, found 190.0997.
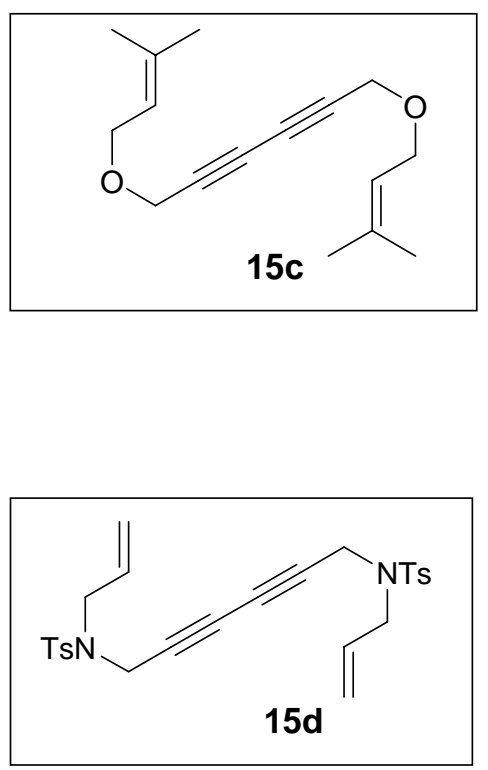
HRMS $\boldsymbol{m} / \mathbf{z}$ (ESI): calc. for $\mathrm{C}_{26} \mathrm{H}_{28} \mathrm{~N}_{2} \mathrm{O}_{4} \mathrm{~S}_{2} \mathrm{Na}\left[\mathrm{M}^{+}+\mathrm{Na}\right] 519.1388$, found 519.1384. calc. for $\mathrm{C}_{16} \mathrm{H}_{22} \mathrm{O}_{2} \mathrm{Na}\left[\mathrm{M}^{+}+\mathrm{Na}\right] 269.1517$, found 269.1519 .

Colorless oil: ${ }^{\mathbf{1}} \mathbf{H}$ NMR (300 MHz, $\mathbf{C D C l}_{3}$ ) $\delta 5.89$ (ddt, $J=17.3$, $10.4,5.8 \mathrm{~Hz}, 2 \mathrm{H}), 5.32(\mathrm{ddt}, J=17.3,1.6,1.4 \mathrm{~Hz}, 2 \mathrm{H}), 5.23$ (ddt, $J=10.4,1.6,1.4 \mathrm{~Hz}, 2 \mathrm{H}), 4.23(\mathrm{~s}, 4 \mathrm{H}), 4.06$ (dt, $J=5.8,1.4 \mathrm{~Hz}$, 4H); ${ }^{13} \mathbf{C}$ NMR (75 MHz, $\left.\mathbf{C D C l}_{3}\right) \delta$ 133.86(2), 118.33(2), 75.53(2), 70.97(2), 70.55(2), 57.72(2); IR (neat): $v_{\max }=2851$, 2137, 1348, $1086 \mathrm{~cm}^{-1}$; HRMS $\mathbf{m} / \mathbf{z}$ (EI): calc. for $\mathrm{C}_{12} \mathrm{H}_{14} \mathrm{O}_{2}\left[\mathrm{M}^{+}\right]$

Colorless oil: ${ }^{1} \mathbf{H}$ NMR (300 MHz, $\mathbf{C D C l}_{3}$ ) $\delta 5.32(\mathrm{t}, J=7.0 \mathrm{~Hz}$, 2H), $4.20(\mathrm{~s}, 4 \mathrm{H}), 4.05(\mathrm{~d}, J=7.0 \mathrm{~Hz}, 4 \mathrm{H}), 1.76(\mathrm{~s}, 6 \mathrm{H}), 1.71$ (s, 6H); ${ }^{13} \mathbf{C}$ NMR (75 $\left.\mathbf{M H z}, \mathbf{C D C l}_{3}\right) \delta$ 138.90(2), 120.20(2), 75.78(2), 70.40(2), 66.34(2), 57.43(2), 25.99(2), 18.24(2); IR (neat): $v_{\max }=2933,2241,1349,1070 \mathrm{~cm}^{-1}$; HRMS $\mathbf{m} / \mathbf{z}$ (ESI):

White solid: ${ }^{1} \mathbf{H}$ NMR (300 MHz, $\left.\mathbf{C D C l}_{3}\right) \delta 7.70(\mathrm{~d}, J=8.2 \mathrm{~Hz}$, 4H), 7.29 (d, $J=8.2 \mathrm{~Hz}, 4 \mathrm{H}), 5.69$ (ddt, $J=17.3,9.8,6.5 \mathrm{~Hz}, 2 \mathrm{H}$ ), 5.28-5.22 (m, 4H), $4.11(\mathrm{~s}, 4 \mathrm{H}), 3.75(\mathrm{~d}, J=6.5 \mathrm{~Hz}, 4 \mathrm{H}), 2.42(\mathrm{~s}$, 6H); ${ }^{13} \mathrm{C}$ NMR (75 MHz, $\left.\mathbf{C D C l}_{3}\right) \delta$ 144.03(2), 135.83(2), 131.83(2), 129.76(4), 127.82(4), 120.37(2), 71.93(2), 69.40(2), 49.55(2), 36.60(2), 21.72(2); IR: $v_{\max } 2915,1343,1163 \mathrm{~cm}^{-1}$; 


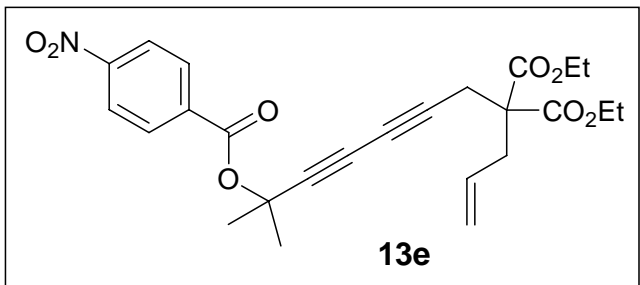

Colorless oil: ${ }^{\mathbf{1}} \mathbf{H}$ NMR (300 MHz, $\left.\mathbf{C D C l}_{3}\right) \delta 8.28(\mathrm{dt}$, $J=8.8,2.0 \mathrm{~Hz}, 2 \mathrm{H}), 8.17(\mathrm{dt}, J=8.8,2.0 \mathrm{~Hz}, 2 \mathrm{H})$, $5.61(\mathrm{ddt}, J=16.9,10.0,7.4 \mathrm{~Hz}, 1 \mathrm{H}), 5.22-5.11(\mathrm{~m}$, $2 \mathrm{H}), 4.21(\mathrm{q}, J=7.2 \mathrm{~Hz}, 4 \mathrm{H}), 2.90(\mathrm{~s}, 2 \mathrm{H}), 2.78(\mathrm{~d}, J=$ $7.4 \mathrm{~Hz}, 2 \mathrm{H}), 1.83(\mathrm{~s}, 6 \mathrm{H}), 1.25(\mathrm{t}, J=7.2 \mathrm{~Hz}, 6 \mathrm{H}) ;{ }^{13} \mathrm{C}$ NMR (75 MHz, $\left.\mathbf{C D C l}_{3}\right) \delta 169.60(2), 162.91,150.73$, 136.29, 131.70, 130.95(2), 123.65(2), 120.29, 76.00, 74.08(2), 70.08, 67.30, 62.02(2), 56.87, 36.85, 28.98(2), 23.76, 14.23(2); IR (neat): $v_{\max }=2985,2256,1733,1278 \mathrm{~cm}^{-1}$; HRMS m/z (ESI): calc. for $\mathrm{C}_{25} \mathrm{H}_{27} \mathrm{NO}_{8} \mathrm{Na}\left[\mathrm{M}^{+}+\mathrm{Na}\right] 492.1634$, found 492.1049 .

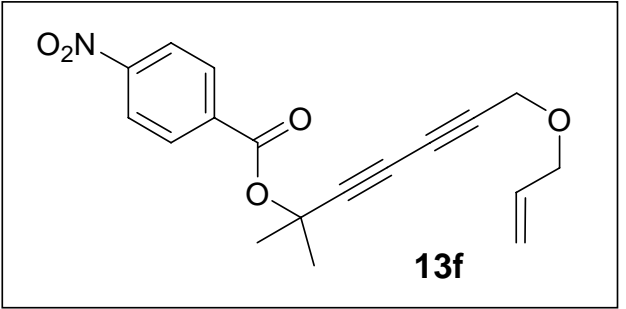

Colorless oil: ${ }^{\mathbf{1}} \mathbf{H}$ NMR (300 MHz, $\mathbf{C D C l}_{3}$ ) $\delta 8.29$ (dt, $J$ $=8.9,2.1 \mathrm{~Hz}, 2 \mathrm{H}), 8.18(\mathrm{dt}, J=8.9,2.1 \mathrm{~Hz}, 2 \mathrm{H}), 5.88$ (ddt, $J=17.0,10.5,5.8 \mathrm{~Hz}, 1 \mathrm{H}), 5.31$ (ddt, $J=17.0,1.6$, $1.2 \mathrm{~Hz}, 1 \mathrm{H}$ ), 5.22 (ddt, $J=10.5,1.6,1.2 \mathrm{~Hz}, 1 \mathrm{H}), 4.23$ (s, 2H), $4.06(\mathrm{dt}, J=5.8,1.2 \mathrm{~Hz}, 2 \mathrm{H}), 1.85(\mathrm{~s}, 6 \mathrm{H}) ;{ }^{13} \mathrm{C}$ NMR (75 MHz, $\left.\mathbf{C D C l}_{3}\right) \delta 162.92,150.75,136.16$, $133.84,130.95(2), 123.67(2), 118.32,78.58,77.44$, 73.91, 70.99, 70.23, 69.55, 57.73, 28.91(2); HRMS m/z (EI): calc. for $\mathrm{C}_{18} \mathrm{H}_{17} \mathrm{NO}_{5}\left[\mathrm{M}^{+}\right] 327.1107$, found 327.1093 .

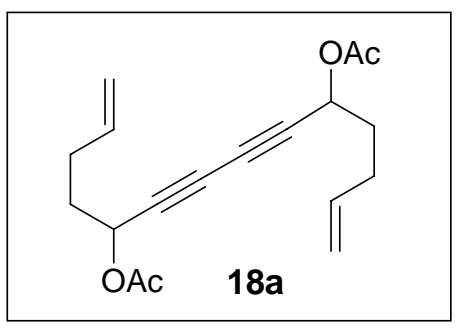

Colorless oil: ${ }^{1} \mathbf{H}$ NMR (300 $\left.\mathbf{M H z}, \mathbf{C D C l}_{3}\right) \delta 5.78$ (ddt, $J=16.8$, 10.1, $6.7 \mathrm{~Hz}, 2 \mathrm{H}), 5.39$ (t, $J=6.7 \mathrm{~Hz}, 2 \mathrm{H}), 5.10-4.99(\mathrm{~m}, 4 \mathrm{H}), 2.20$ $(\mathrm{dt}, J=7.9,6.7 \mathrm{~Hz}, 4 \mathrm{H}), 2.08(\mathrm{~s}, 6 \mathrm{H}), 1.92-1.84(\mathrm{~m}, 4 \mathrm{H}) ;{ }^{13} \mathrm{C}$ NMR (75 MHz, $\left.\mathbf{C D C l}_{3}\right) \delta$ 169.91(2), 136.76(2), 116.08(2), 77.03(2), 69.61(2), 63.73(2), 33.78(2), 29.26(2), 21.04(2); IR (neat): $v_{\max }=2937,2166,1747,1227 \mathrm{~cm}^{-1}$; HRMS m/z (ESI): calc. for $\mathrm{C}_{18} \mathrm{H}_{22} \mathrm{O}_{4} \mathrm{Na}\left[\mathrm{M}^{+}+\mathrm{Na}\right] 325.1416$, found 325.1422.

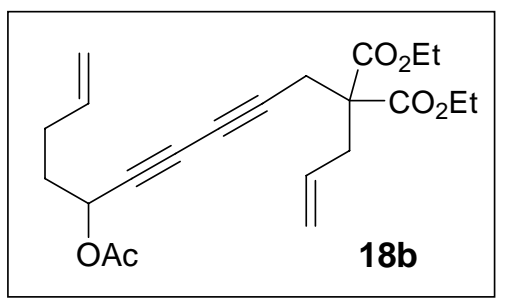

Colorless oil: ${ }^{1} \mathbf{H}$ NMR (300 MHz, $\left.\mathbf{C D C l}_{3}\right) \delta$ 5.85-5.71 (m, 1H), 5.68-5.54 (m, 1H), 5.39 (t, $J=6.5 \mathrm{~Hz}, 1 \mathrm{H}), 5.22-4.99(\mathrm{~m}, 4 \mathrm{H})$, $4.21(\mathrm{q}, J=7.2 \mathrm{~Hz}, 4 \mathrm{H}), 2.89$ (s, 2H), 2.78 (d, $J=7.4 \mathrm{~Hz}, 2 \mathrm{H})$, $2.19(\mathrm{q}, J=6.8 \mathrm{~Hz}, 2 \mathrm{H}), 2.08(\mathrm{~s}, 3 \mathrm{H}), 1.90-1.82(\mathrm{~m}, 2 \mathrm{H}), 1.26(\mathrm{t}$, $J=7.2 \mathrm{~Hz}, 3 \mathrm{H}) ;{ }^{13} \mathbf{C}$ NMR (75 $\left.\mathbf{M H z}, \mathbf{C D C l}_{3}\right) \delta 169.89$, 169.58(2), 136.85, 131.71, 120.28, 115.93, 76.22, 73.60, 70.34, $67.32,63.78,62.01(2), 56.87,36.85,33.90,29.27,23.70,21.06$ 14.23(2); IR (neat): $v_{\max }=2983,2260,1737,1218 \mathrm{~cm}^{-1}$; HRMS m/z (EI): calc. for $\mathrm{C}_{22} \mathrm{H}_{28} \mathrm{O}_{6}\left[\mathrm{M}^{+}\right]$ 388.1886 , found 388.1868 . 


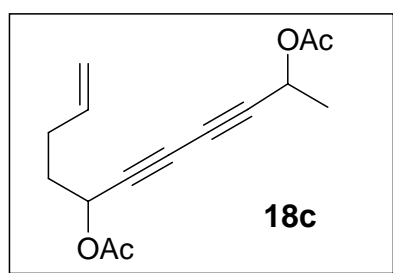

Colorless oil: ${ }^{1} \mathbf{H}$ NMR (300 MHz, $\mathbf{C D C l}_{3}$ ) $\delta 5.78$ (ddt, $J=16.9,10.1$, $6.7 \mathrm{~Hz}, 1 \mathrm{H}), 5.46$ (q, $J=6.9 \mathrm{~Hz}, 1 \mathrm{H}), 5.39$ (t, $J=6.9 \mathrm{~Hz}, 1 \mathrm{H}), 5.10-$ $4.99(\mathrm{~m}, 2 \mathrm{H}), 2.20(\mathrm{td}, J=7.3,6.7 \mathrm{~Hz}, 2 \mathrm{H}), 2.08(\mathrm{~s}, 3 \mathrm{H}), 2.07(\mathrm{~s}, 3 \mathrm{H})$, 1.91-1.83 (m, 2H), $1.50(\mathrm{~d}, J=6.9 \mathrm{~Hz}, 3 \mathrm{H}) ;{ }^{13} \mathbf{C}$ NMR (75 MHz, $\left.\mathbf{C D C l}_{3}\right) \delta 169.86,169.84,136.74,116.04,77.95,77.08,69.55,68.78$, $63.69,60.45,33.75,29.22,33.75,29.22,21.08,21.06,21.01$; HRMS $\mathbf{m} / \mathbf{z}$ (ESI): calc. for $\mathrm{C}_{15} \mathrm{H}_{18} \mathrm{O}_{4} \mathrm{Na}\left[\mathrm{M}^{+}+\mathrm{Na}\right] 285.1103$, found 285.1097 .

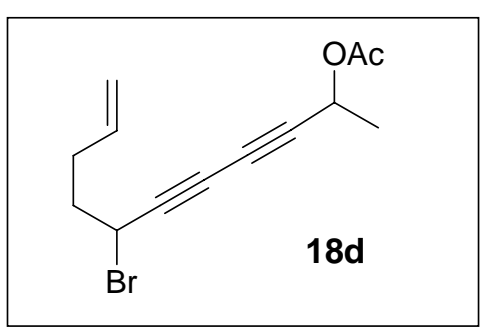

305.0153, found 305.0158.

Colorless oil: ${ }^{1} \mathbf{H}$ NMR (300 MHz, $\mathbf{C D C l}_{3}$ ) $\delta 5.76$ (ddt, $J=16.9$, $10.1,6.6 \mathrm{~Hz}, 1 \mathrm{H}), 5.48(\mathrm{q}, J=6.8 \mathrm{~Hz}, 1 \mathrm{H}), 5.14-5.03(\mathrm{~m}, 2 \mathrm{H})$, $4.53(\mathrm{t}, J=6.8 \mathrm{~Hz}, 1 \mathrm{H}), 2.33-2.25(\mathrm{~m}, 2 \mathrm{H}), 2.14-2.06(\mathrm{~m}, 2 \mathrm{H})$, $2.08(\mathrm{~s}, 3 \mathrm{H}), 1.51(\mathrm{~d}, J=6.8 \mathrm{~Hz}, 3 \mathrm{H}) ;{ }^{13} \mathbf{C} \mathbf{N M R}\left(75 \mathbf{~ M H z}, \mathbf{C D C l}_{3}\right)$ $\delta$ 169.83, 136.01, 116.70, 79.64, 77.75, 70.88, 68.70, 60.48, 38.40, 35.51, 31.44, 21.07(2); IR (neat): $v_{\max }=2938,2163,1746$, $1228 \mathrm{~cm}^{-1}$; HRMS $\mathbf{m} / \mathbf{z}$ (ESI): calc. for $\mathrm{C}_{13} \mathrm{H}_{15} \mathrm{BrO}_{2} \mathrm{Na}\left[\mathrm{M}^{+}+\mathrm{Na}\right]$

\section{General procedures for $\mathrm{PtCl}_{2}$-catalyzed reactions}

$\mathrm{PtCl}_{2}(5 \mathrm{~mol} \%)$ was added to a solution of the diyne in toluene $(0.03 \mathrm{M})$, and $\mathrm{CO}$ was bubbled through the solution. The mixture was warmed to $80{ }^{\circ} \mathrm{C}$ and stirred until the reaction was complete $(2 \sim 15 \mathrm{hrs})$. The mixture was cooled to room temperature and the solvent was evaporated under vacuum. Purification by flash chromatography on silica gel (hexanes/ethyl ether) afforded the product.

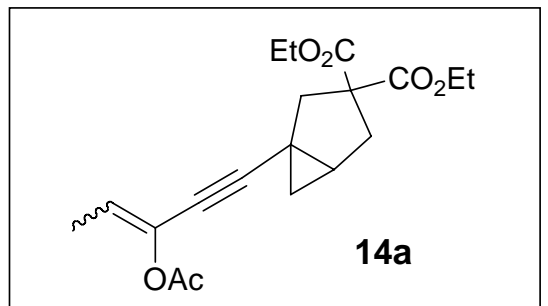

Colorless oil ( $88 \%$ yield, Crude $Z: E=2.5: 1$, after column $Z: E$ $=1: 3.3$ ): ${ }^{1} \mathbf{H}$ NMR (300 MHz, $\left.\mathbf{C D C l}_{3}\right) \boldsymbol{E}$-isomer $\delta 5.63$ (q, $J$ $=7.2 \mathrm{~Hz}, 1 \mathrm{H}), 4.15(\mathrm{q}, J=7.1 \mathrm{~Hz}, 4 \mathrm{H}), 2.83(\mathrm{~d}, J=13.8 \mathrm{~Hz}$, $1 \mathrm{H}), 2.63-2.52(\mathrm{~m}, 3 \mathrm{H}), 2.14(\mathrm{~s}, 3 \mathrm{H}), 1.81(\mathrm{~d}, J=7.2 \mathrm{~Hz}, 3 \mathrm{H})$, $1.80-1.72(\mathrm{~m} .1 \mathrm{H}), 1.27-1.20(\mathrm{~m}, 6 \mathrm{H}), 1.01-0.93(\mathrm{~m}, 1 \mathrm{H})$, $0.67(\mathrm{dd}, J=5.4,5.4 \mathrm{~Hz}, 1 \mathrm{H})$; $Z$ - isomer $\delta 5.65(\mathrm{q}, J=7.2$ $\mathrm{Hz}, 1 \mathrm{H}), 4.15(\mathrm{q}, J=7.1 \mathrm{~Hz}, 4 \mathrm{H}), 2.80(\mathrm{~d}, J=13.8 \mathrm{~Hz}, 1 \mathrm{H})$, 2.63-2.52 (m, 3H), $2.19(\mathrm{~s}, 3 \mathrm{H}), 1.60(\mathrm{~d}, J=7.2 \mathrm{~Hz}, 3 \mathrm{H}), 1.80-1.72(\mathrm{~m}, 1 \mathrm{H}), 1.27-1.20(\mathrm{~m}, 6 \mathrm{H})$, 1.01-0.93 (m, 1H), $0.62(\mathrm{dd}, J=5.4,5.4 \mathrm{~Hz}, 1 \mathrm{H}) ;{ }^{13} \mathbf{C}$ NMR (75 MHz, $\left.\mathbf{C D C l}_{3}\right) \boldsymbol{E}$-isomer $\delta 172.51$, $171.23,169.34,131.14,123.10,98.25,70.27,62.15,61.99,59.71,39.99,35.73,28.47,21.08,19.20$, 18.92, 14.19(2), 13.60; $\boldsymbol{Z}$-isomer $\delta 172.51,171.23,169.34,130.86,122.80,98.25,69.89,62.15$, $61.99,59.71,39.94,35.73,28.24,20.84,18.92,18.71,14.19(2), 11.78$; IR (neat): $v_{\max }=2921$, 2219, 1731, 1445, 1367, $1250 \mathrm{~cm}^{-1}$; HRMS m/z (EI): calc. for $\mathrm{C}_{19} \mathrm{H}_{24} \mathrm{O}_{6}\left[\mathrm{M}^{+}\right]$348.1573, found 


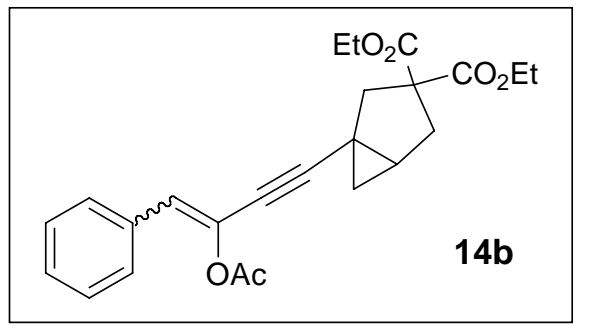

Colorless oil (92\% yield, Z:E=6.5:1): ${ }^{1} \mathbf{H}$ NMR (300 MHz, $\left.\mathbf{C D C l}_{3}\right) \mathrm{Z}$ - isomer $\delta$ 7.42-7.22 (m, 5H), $6.39(\mathrm{~s}, 1 \mathrm{H}), 4.18$ (q, $J=7.1 \mathrm{~Hz}, 2 \mathrm{H}), 4.16(\mathrm{q}, J=7.1 \mathrm{~Hz}, 2 \mathrm{H}), 2.84(\mathrm{~d}, J=$ $13.6 \mathrm{~Hz}, 1 \mathrm{H}), 2.64-2.57(\mathrm{~m}, 3 \mathrm{H}), 2.24(\mathrm{~s}, 3 \mathrm{H}), 1.85-1.78(\mathrm{~m}$, $1 \mathrm{H}), 1.25(\mathrm{t}, J=7.1 \mathrm{~Hz}, 3 \mathrm{H}), 1.23(\mathrm{t}, J=7.1 \mathrm{~Hz}, 3 \mathrm{H}), 1.02$ $(\mathrm{dd}, J=8.4,5.8 \mathrm{~Hz}, 1 \mathrm{H}), 0.68(\mathrm{dd}, J=5.8,5.8 \mathrm{~Hz}, 1 \mathrm{H}) ;{ }^{13} \mathrm{C}$ NMR (75 $\mathbf{M H z}, \mathbf{C D C l}_{3}$ ) Z- isomer $\delta$ 172.41, 171.11, 167.97, 133.79, 129.91, 128.97(2), 128.67(2), 128.40, 128.25, 125.01, 94.90, 73.53, 62.08, 61.93, 59.63, 39.83, 35.66, 28.48, 21.19, 19.09, 18.84, 14.13; IR (neat): $v_{\max }=2982,2214,1730,1196$ $\mathrm{cm}^{-1} ; \mathbf{H R M S ~ m / z ~ ( E I ) : ~ c a l c . ~ f o r ~} \mathrm{C}_{24} \mathrm{H}_{26} \mathrm{O}_{6}\left[\mathrm{M}^{+}\right] 410.1729$, found 410.1718 .

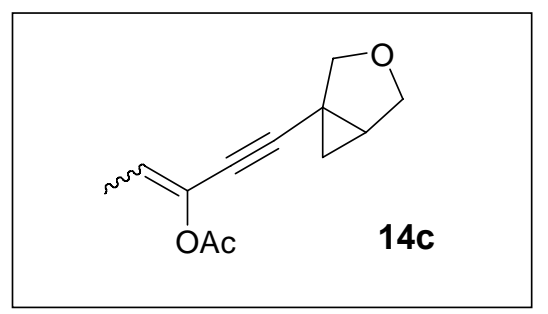

Colorless oil $(87 \%$ yield, $Z: E=2.7: 1$ after column $Z: E$ $=1.1: 1):{ }^{1} \mathbf{H}$ NMR (300 MHz, $\mathbf{C D C l}_{3}$ ) Z- isomer $\delta 5.69$ (q, $J$ $=7.2 \mathrm{~Hz}, 1 \mathrm{H}), 3.86(\mathrm{~d}, J=8.1 \mathrm{~Hz}, 1 \mathrm{H}), 3.79(\mathrm{~m}, 2 \mathrm{H}), 3.69(\mathrm{~d}$, $J=8.1 \mathrm{~Hz}, 1 \mathrm{H}), 2.20(\mathrm{~s}, 3 \mathrm{H}), 1.97-1.89(\mathrm{~m}, 1 \mathrm{H}), 1.61(\mathrm{~d}, J=$ $7.2 \mathrm{~Hz}, 3 \mathrm{H}), 1.11-1.05$ (m, 1H), 0.94 (dd, $J=4.9,4.9 \mathrm{~Hz}, 1 \mathrm{H})$; $\boldsymbol{E}$ - isomer $\delta 5.67(\mathrm{q}, J=7.2 \mathrm{~Hz}, 1 \mathrm{H}), 3.90(\mathrm{~d}, J=8.1 \mathrm{~Hz}, 1 \mathrm{H})$, 3.81(m, 2H), $3.72(\mathrm{~d}, J=8.1 \mathrm{~Hz}, 1 \mathrm{H}), 2.15(\mathrm{~s}, 3 \mathrm{H}), 1.97-1.89$ (m. 1H), $1.83(\mathrm{~d}, J=7.2 \mathrm{~Hz}, 3 \mathrm{H}), 1.11-1.05(\mathrm{~m}, 1 \mathrm{H}), 0.98(\mathrm{dd}, J=4.9,4.9 \mathrm{~Hz}, 1 \mathrm{H}) ;{ }^{13} \mathbf{C} \mathbf{N M R}(\mathbf{7 5}$ MHz, $\mathbf{C D C l}_{3}$ ) Z-isomer $\delta 168.30,130.64,123.24,88.96,73.88,71.66,69.46,27.75,20.73,18.64$, $15.85,11.72$; $\boldsymbol{E}$-isomer $\delta 169.22,130.91,123.51,88.96,73.88,71.66,69.46,27.99,20.97,18.85$, 16.06, 13.54; IR (neat): $v_{\max }=2932,2869,2215,1714,1587,1202,1086,911 \mathrm{~cm}^{-1} ;$ HRMS m/z (EI): calc. for $\mathrm{C}_{12} \mathrm{H}_{14} \mathrm{O}_{3}\left[\mathrm{M}^{+}\right]$206.0943, found 206.0946.

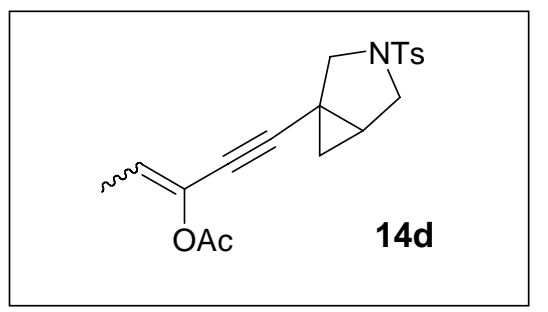

Colorless oil (82\% yield, Z:E=4.7:1): ${ }^{1} \mathbf{H}$ NMR (300 MHz, $\left.\mathbf{C D C l}_{3}\right) \mathrm{Z}$ - isomer $\delta 7.66(\mathrm{~d}, J=8.0 \mathrm{~Hz}, 2 \mathrm{H}), 7.34(\mathrm{~d}, J=8.0$ $\mathrm{Hz}, 2 \mathrm{H}), 5.65$ (q, $J=7.2 \mathrm{~Hz}, 1 \mathrm{H}), 3.64(\mathrm{~d}, J=9.2 \mathrm{~Hz}, 1 \mathrm{H})$, $3.52(\mathrm{~d}, J=9.2 \mathrm{~Hz}, 1 \mathrm{H}), 3.09(\mathrm{dd}, J=9.2,3.8 \mathrm{~Hz}, 1 \mathrm{H}), 3.02$ $(\mathrm{d}, J=9.2 \mathrm{~Hz}, 1 \mathrm{H}), 2.45(\mathrm{~s}, 3 \mathrm{H}), 2.16(\mathrm{~s}, 3 \mathrm{H}), 1.80-1.74(\mathrm{~m}$, 1H), $1.58(\mathrm{~d}, J=7.2 \mathrm{~Hz}, 3 \mathrm{H}), 1.11-1.02(\mathrm{~m}, 2 \mathrm{H}) ;{ }^{13} \mathbf{C}$ NMR $\left(75 \mathbf{~ M H z}, \mathbf{C D C l}_{3}\right) \mathrm{Z}$ - isomer $\delta$ 168.26, 143.95, 133.22, 130.30, 129.92(2), 127.72(2), 123.79, 88.60, 74.04, 52.24, 49.42, 26.19, 21.66, 20.69, 17.58, 16.79, 11.71; IR (neat): $v_{\max }=2933,2216,1761,1167 \mathrm{~cm}^{-1}$; HRMS $\mathbf{m} / \mathbf{z}$ (ESI): calc. for $\mathrm{C}_{19} \mathrm{H}_{21} \mathrm{NO}_{4} \mathrm{SNa}$ $\left[\mathrm{M}^{+}+\mathrm{Na}\right]$ 382.1089, found 382.1093. 


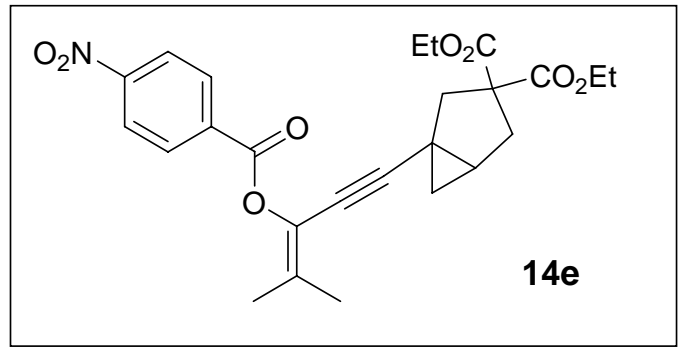

Colorless oil (98\% yield): ${ }^{1} \mathbf{H}$ NMR (300 MHz, $\left.\mathbf{C D C l}_{3}\right) \delta 8.35-8.28(\mathrm{~m}, 4 \mathrm{H}), 4.17(\mathrm{q}, J=7.1 \mathrm{~Hz}$, 2H), $4.14(\mathrm{q}, J=7.1 \mathrm{~Hz}, 2 \mathrm{H}), 2.82(\mathrm{~d}, J=13.5 \mathrm{~Hz}$, $1 \mathrm{H}), 2.61-2.55(\mathrm{~m}, 3 \mathrm{H}), 1.98(\mathrm{~s}, 3 \mathrm{H}), 1.80-1.74(\mathrm{~m}$, $1 \mathrm{H}), 1.71(\mathrm{~s}, 3 \mathrm{H}), 1.24(\mathrm{t}, J=7.1 \mathrm{~Hz}, 3 \mathrm{H}), 1.21(\mathrm{t}, J$ $=7.1 \mathrm{~Hz}, 3 \mathrm{H}), 1.00-0.95(\mathrm{~m} .1 \mathrm{H}), 0.65(\mathrm{dd}, J=5.4$, $5.4 \mathrm{~Hz}, 1 \mathrm{H}) ;{ }^{13} \mathbf{C}$ NMR (75 MHz, $\left.\mathbf{C D C l}_{3}\right) \delta 172.45$, $171.20,162.59,150.96,135.17,132.53,131.40(2), 125.88,123.79(2), 97.19,70.91,62.08,61.93$, 59.64, 40.02, 35.67, 28.37, 20.80, 19.14, 18.91, 17.80, 14.13(2); IR (neat): $v_{\max }=2983,2218,1732$, 1531, 1259, $1115 \mathrm{~cm}^{-1}$; HRMS $\mathbf{m} / \mathbf{z}$ (ESI): calc. for $\mathrm{C}_{25} \mathrm{H}_{27} \mathrm{NO}_{8} \mathrm{Na}\left[\mathrm{M}^{+}+\mathrm{Na}\right]$ 492.1634, found 492.1620 .

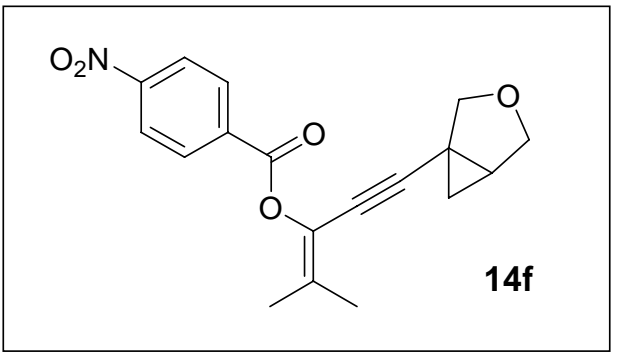

Colorless oil (90\% yield): ${ }^{1} \mathbf{H}$ NMR (300 $\mathbf{M H z}, \mathbf{C D C l}_{3}$ ) $\delta$ 8.35-8.28 (m, 4H), $3.89(\mathrm{~d}, J=7.8 \mathrm{~Hz}, 1 \mathrm{H}), 3.81-3.80$ $(\mathrm{m}, 2 \mathrm{H}), 3.71(\mathrm{dd}, J=7.8,0.3 \mathrm{~Hz}, 1 \mathrm{H}), 1.99(\mathrm{~s}, 3 \mathrm{H})$, $1.95-1.90(\mathrm{~m}, 1 \mathrm{H}), 1.73(\mathrm{~s}, 3 \mathrm{H}), 1.09$ (dd, $J=8.1,4.8 \mathrm{~Hz}$, 1H), $0.96(\mathrm{dd}, J=4.8,4.8 \mathrm{~Hz}, 1 \mathrm{H}) ;{ }^{13} \mathrm{C}$ NMR (75 MHz, $\left.\mathbf{C D C l}_{3}\right) \delta 162.61,151.02,135.09,133.18,131.41(2)$, $125.75,123.84,93.80,72.35,71.81,69.52,27.98,20.85$, 18.92, 17.86, 16.10; IR (neat): $v_{\max }=2860,2220,1742,1530,1267,1116 \mathrm{~cm}^{-1} ; \mathbf{H R M S ~ m / z ~ ( E I ) : ~}$ calc. for $\mathrm{C}_{18} \mathrm{H}_{17} \mathrm{NO}_{5}\left[\mathrm{M}^{+}\right] 327.1107$, found 327.1093 .

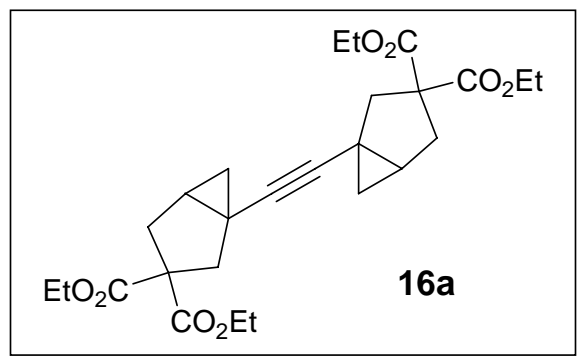

Colorless oil (96\% yield): ${ }^{1} \mathbf{H}$ NMR (300 $\left.\mathbf{M H z}, \mathbf{C D C l}_{3}\right) \delta$ 4.17 (q, $J=7.2 \mathrm{~Hz}, 4 \mathrm{H}), 4.14$ (q, $J=7.2 \mathrm{~Hz}, 4 \mathrm{H}), 2.75(\mathrm{~d}, J$ $=13.6 \mathrm{~Hz}, 2 \mathrm{H}), 2.54-2.48(\mathrm{~m}, 6 \mathrm{H}), 1.64-1.58(\mathrm{~m}, 2 \mathrm{H}), 1.23$ $(\mathrm{t}, J=7.2 \mathrm{~Hz}, 6 \mathrm{H}), 1.22(\mathrm{t}, J=7.2 \mathrm{~Hz}, 6 \mathrm{H}), 0.82(\mathrm{dd}, J=$ 8.5, $5.5 \mathrm{~Hz}, 2 \mathrm{H}), 0.51(\mathrm{dd}, J=5.5,5.1 \mathrm{~Hz}, 2 \mathrm{H}) ;{ }^{13} \mathbf{C}$ NMR (75 MHz, $\left.\mathbf{C D C l}_{3}\right) \delta$ 172.62(2), 171.42(2), 80.60(2), 62.02(2), 61.86(2), 59.63(2), 40.61(2), 35.76(2), 27.51(2), 18.66(2), 18.43(2), 14.20(2), 14.17(2); IR (neat): $v_{\max }=$ 2982, 2080, 1729, $1249 \mathrm{~cm}^{-1}$; HRMS m/z (ESI): calc. for $\mathrm{C}_{26} \mathrm{H}_{34} \mathrm{O}_{8} \mathrm{Na}\left[\mathrm{M}^{+}+\mathrm{Na}\right]$ 497.2151, found 497.2142 .

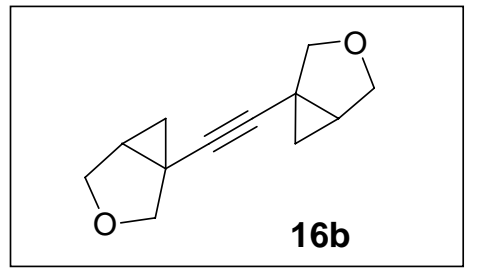

Colorless oil (6.3\% yield): ${ }^{\mathbf{1}} \mathbf{H}$ NMR (300 $\left.\mathbf{M H z}, \mathbf{C D C l}_{3}\right) \delta 3.83$ (d, $J=7.8 \mathrm{~Hz}, 2 \mathrm{H}), 3.79-3.78$ (m, 4H), 3.66 (d, $J=7.8 \mathrm{~Hz}, 2 \mathrm{H}), 1.82-$ $1.77(\mathrm{~m}, 2 \mathrm{H}), 0.97(\mathrm{dd}, J=8.0,4.8 \mathrm{~Hz}, 2 \mathrm{H}), 0.85(\mathrm{dd}, J=4.8,4.8$ $\mathrm{Hz}, 2 \mathrm{H}) ;{ }^{13} \mathbf{C}$ NMR (75 MHz, $\left.\mathbf{C D C l}_{3}\right) \delta$ 78.72(2), 72.27(2), 
69.62(2), 27.20(2), 18.68(2), 15.62(2); IR (neat): $v_{\max }=2924,2854,2230,1017 \mathrm{~cm}^{-1} ;$ HRMS $\mathbf{m} / \mathbf{z}$ (EI): calc. for $\mathrm{C}_{12} \mathrm{H}_{14} \mathrm{O}_{2}\left[\mathrm{M}^{+}\right]$190.0994, found 190.0997.
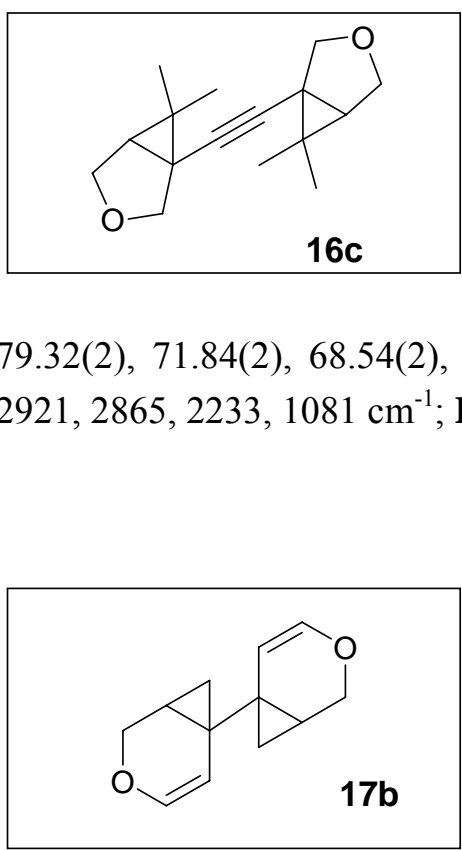

Colorless oil (25\% yield, 1:1 mixture of diastereomers): ${ }^{1} \mathbf{H}$ NMR (300 MHz, $\mathbf{C D C l}_{3}$ ) major $\delta 3.99(\mathrm{dd}, J=4.1,1.6 \mathrm{~Hz}, 2 \mathrm{H}), 3.94-$ $3.80(\mathrm{~m}, 6 \mathrm{H}), 1.56-1.54(\mathrm{~m}, 2 \mathrm{H}), 1.11(\mathrm{~s}, 12 \mathrm{H})$; minor $\delta 4.02(\mathrm{dd}$, $J=4.1,1.6 \mathrm{~Hz}, 2 \mathrm{H}), 3.94-3.80(\mathrm{~m}, 6 \mathrm{H}), 1.56-1.54(\mathrm{~m}, 2 \mathrm{H}), 1.20$ (s, 12H); ${ }^{13} \mathbf{C}$ NMR (75 MHz, $\mathbf{C D C l}_{3}$ ) major $\delta$ 79.25(2), 71.78(2), 68.54(2), 38.47(2), 29.68(2), 25.43(2), 24.57(2), 13.25(2); minor $\delta$ 79.32(2), 71.84(2), 68.54(2), 38.57(2), 29.92(2), 25.62(2), 24.66(2), 13.29(2); IR (neat): $v_{\max }=$ 2921, 2865, 2233, $1081 \mathrm{~cm}^{-1}$; HRMS m/z (EI): calc. for $\mathrm{C}_{16} \mathrm{H}_{22} \mathrm{O}_{2}\left[\mathrm{M}^{+}\right] 246.1620$, found 246.1624 .

Colorless oil (23\% yield, 1.1:1 mixture of diastereomers): ${ }^{1} \mathbf{H}$ NMR (300 MHz, $\mathbf{C D C l}_{3}$ ) major $\delta 6.19(\mathrm{~d}, J=6.0 \mathrm{~Hz}, 2 \mathrm{H}), 5.27$ $(\mathrm{d}, J=6.0 \mathrm{~Hz}, 2 \mathrm{H}), 4.07(\mathrm{dd}, J=10.5,1.2 \mathrm{~Hz}, 2 \mathrm{H}), 3.72(\mathrm{dd}, J=$ $10.5,2.0 \mathrm{~Hz}, 2 \mathrm{H}), 1.26-1.22(\mathrm{~m}, 2 \mathrm{H}), 0.85-0.71(\mathrm{~m}, 4 \mathrm{H})$; minor $\delta$ $6.18(\mathrm{~d}, J=6.0 \mathrm{~Hz}, 2 \mathrm{H}), 5.24(\mathrm{~d}, J=6.0 \mathrm{~Hz}, 2 \mathrm{H}), 4.10$ (dd, $J=$ $10.5,1.2 \mathrm{~Hz}, 2 \mathrm{H}), 3.79(\mathrm{dd}, J=10.5,2.0 \mathrm{~Hz}, 2 \mathrm{H}), 1.37-1.32(\mathrm{~m}$, 2H), 0.85-0.71 (m, 4H); ${ }^{13} \mathbf{C}$ NMR (75 MHz, $\mathbf{C D C l}_{3}$ ) major $\delta$ 141.77(2), 108.91(2), 62.59(2), 22.76(2), 19.98(2), 17.14(2); minor $\delta$ 141.59(2), 109.28(2), 62.65(2), 22.92(2), 19.92(2), 17.00(2); IR (neat): $v_{\max }=2865,1639,1232,1018 \mathrm{~cm}^{-1}$; HRMS m/z (EI): calc. for $\mathrm{C}_{12} \mathrm{H}_{14} \mathrm{O}_{2}\left[\mathrm{M}^{+}\right] 190.0994$, found 190.0994 .

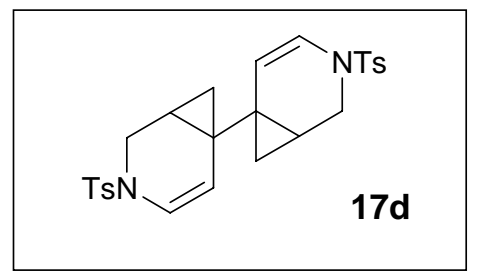

White solid (63\% yield, 1.3:1 mixture of diastereomers): ${ }^{1} \mathbf{H}$ NMR (300 MHz, $\mathbf{C D C l}_{3}$ ) major $\delta 7.62(\mathrm{~d}, J=8.5 \mathrm{~Hz}, 4 \mathrm{H}), 7.30(\mathrm{~d}, J=$ $8.5 \mathrm{~Hz}, 4 \mathrm{H}), 6.35(\mathrm{~d}, J=8.2 \mathrm{~Hz}, 2 \mathrm{H}), 5.29(\mathrm{~d}, J=8.2 \mathrm{~Hz}, 2 \mathrm{H})$, $3.81(\mathrm{~d}, J=11.6 \mathrm{~Hz}, 2 \mathrm{H}), 2.88(\mathrm{dd}, J=11.6,2.6 \mathrm{~Hz}, 2 \mathrm{H}), 2.42$ (s, $6 \mathrm{H}), 1.22-1.16(\mathrm{~m}, 2 \mathrm{H}), 0.58(\mathrm{dd}, J=9.0,4.8 \mathrm{~Hz}, 2 \mathrm{H}), 0.40$ (dd, $J$ $=5.2,4.8 \mathrm{~Hz}, 2 \mathrm{H})$; minor $\delta 7.62(\mathrm{~d}, J=8.5 \mathrm{~Hz}, 4 \mathrm{H}), 7.30(\mathrm{~d}, J=$ $8.5 \mathrm{~Hz}, 4 \mathrm{H}), 6.33$ (d, $J=8.2 \mathrm{~Hz}, 2 \mathrm{H}), 5.20(\mathrm{~d}, J=8.2 \mathrm{~Hz}, 2 \mathrm{H}), 3.87$ (d, $J=11.6 \mathrm{~Hz}, 2 \mathrm{H}), 2.96(\mathrm{dd}, J$ $=11.6,2.6 \mathrm{~Hz}, 2 \mathrm{H}), 2.42(\mathrm{~s}, 6 \mathrm{H}), 1.22-1.16(\mathrm{~m}, 2 \mathrm{H}), 0.48(\mathrm{dd}, J=9.0,4.8 \mathrm{~Hz}, 2 \mathrm{H}), 0.31(\mathrm{dd}, J=5.2$, $4.8 \mathrm{~Hz}, 2 \mathrm{H}) ;{ }^{13} \mathbf{C}$ NMR (75 MHz, $\mathbf{C D C l}_{3}$ ) major $\delta$ 143.98(2), 135.11(2), 129.99(4), 127.23(4), 121.86(2), 114.25(2), 41.05(2), 29.91(2), 23.76(2), 21.75(2), 17.61(2); minor $\delta$ 143.98(2), 135.11(2), 129.99(4), 127.23(4), 121.74(2), 114.64(2), 41.14(2), 24.13(2), 21.75(2), 21.28(2), 17.16(2); HRMS m/z (ESI): calc. for $\mathrm{C}_{26} \mathrm{H}_{28} \mathrm{~N}_{2} \mathrm{O}_{4} \mathrm{~S}_{2} \mathrm{Na}\left[\mathrm{M}^{+}+\mathrm{Na}\right] 519.1388$, found 519.1375. 


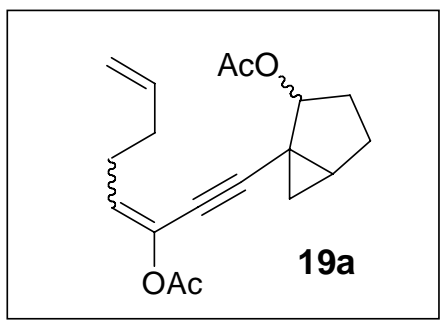

$91.40,80.30,79.47,73.33,73.11,71.16,33.11,32.60,29.81,28.37,28.22,27.42,26.48,26.38$, $25.73,24.82,21.77,21.55,21.38,21.32,21.08,20.87,15.34,15.17$; IR (neat): $v_{\max }=2920,2221$, 1763, 1739, 1371, $1238 \mathrm{~cm}^{-1}$; HRMS m/z (EI): calc. for $\mathrm{C}_{18} \mathrm{H}_{22} \mathrm{O}_{4}\left[\mathrm{M}^{+}\right] 302.1518$, found 302.1531 .

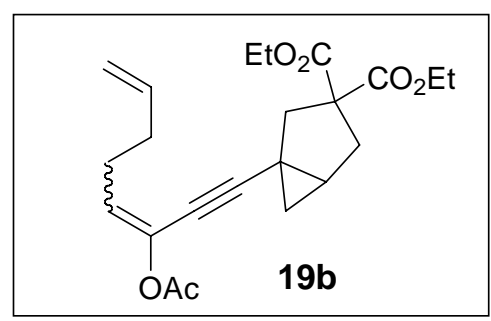

Colorless oil (67\% yield): ${ }^{\mathbf{H}} \mathbf{H}$ NMR (300 $\left.\mathbf{M H z}, \mathbf{C D C l}_{3}\right) \delta 5.89$ $5.59(\mathrm{~m}, 1 \mathrm{H}), 5.59$ (t, $J=7.7 \mathrm{~Hz}, 1 \mathrm{H}), 5.09-4.97(\mathrm{~m}, 2 \mathrm{H}), 4.16$ (q, $J=7.2 \mathrm{~Hz}, 2 \mathrm{H}), 4.15(\mathrm{q}, J=7.2 \mathrm{~Hz}, 2 \mathrm{H}), 2.83(\mathrm{~d}, J=13.8 \mathrm{~Hz}$, $1 \mathrm{H}), 2.61-2.54(\mathrm{~m}, 3 \mathrm{H}), 2.37-2.27(\mathrm{~m}, 1 \mathrm{H}), 2.31(\mathrm{~d}, J=13.8 \mathrm{~Hz}$, $1 \mathrm{H}), 2.21-2.09(\mathrm{~m}, 2 \mathrm{H}), 2.14(\mathrm{~s}, 3 \mathrm{H}), 1.87-1.74(\mathrm{~m}, 1 \mathrm{H}), 1.24(\mathrm{t}$, $J=7.2 \mathrm{~Hz}, 3 \mathrm{H}), 1.23$ (t, $J=7.2 \mathrm{~Hz}, 3 \mathrm{H}), 1.00-0.93(\mathrm{~m}, 1 \mathrm{H}), 0.66$ (dd, $J=5.3,5.3 \mathrm{~Hz}, 1 \mathrm{H}$ ); IR (neat): $v_{\max }=2981,2220,1732$, 1250, $1199 \mathrm{~cm}^{-1}$; HRMS $\mathbf{m} / \mathbf{z}$ (EI): calc. for $\mathrm{C}_{22} \mathrm{H}_{28} \mathrm{O}_{6}\left[\mathrm{M}^{+}\right] 388.1886$, found 388.1868 .

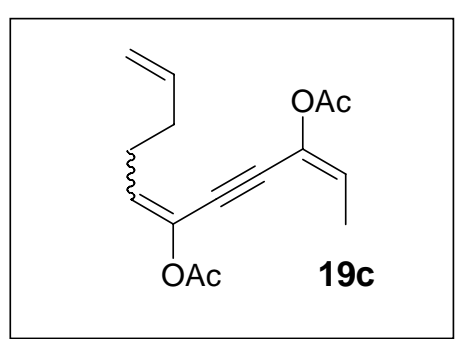

Colorless oil (33\% yield, Z:E=3.5:1): ${ }^{1} \mathbf{H}$ NMR (300 $\mathbf{~ M H z , ~} \mathbf{C D C l}_{3}$ ) $\delta 5.86-5.74(\mathrm{~m}, 1 \mathrm{H}), 5.78(\mathrm{q}, J=7.2 \mathrm{~Hz}, 1 \mathrm{H}), 5.78(\mathrm{t}, J=7.2 \mathrm{~Hz}$, $1 \mathrm{H}), 5.10-4.98(\mathrm{~m}, 2 \mathrm{H}), 2.42-2.13(\mathrm{~m}, 4 \mathrm{H}), 2.19(\mathrm{~s}, 3 \mathrm{H}), 2.15(\mathrm{~s}$, 3H), 1.85 (d, $J=7.2 \mathrm{~Hz}, 3 \mathrm{H}) ;{ }^{13} \mathbf{C}$ NMR (75 $\left.\mathbf{M H z}, \mathbf{C D C l}_{3}\right) \delta$ $169.10,168.16,137.38,130.46,129.68,129.56,125.84,115.78$, $88.39,81.00,32.54,25.84,20.95,20.79,13.70$; IR (neat): $v_{\max }=$ 2920, 2033, 1765, $1188 \mathrm{~cm}^{-1}$; HRMS m/z (EI): calc. for $\mathrm{C}_{15} \mathrm{H}_{18} \mathrm{O}_{4}$

$\left[\mathrm{M}^{+}\right] 262.1205$, found 262.1215.

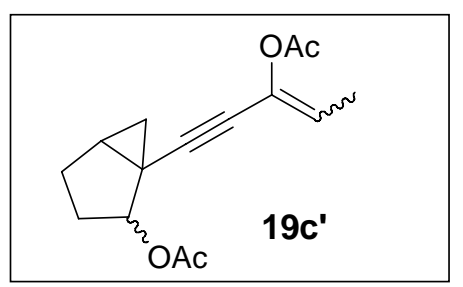

Colorless oil (57\% yield, Z:E=2.2:1): ${ }^{\mathbf{1}} \mathbf{H}$ NMR (300 $\mathbf{M H z}, \mathbf{C D C l}_{3}$ ) $\delta 5.64(\mathrm{q}, J=7.2 \mathrm{~Hz}, 1 \mathrm{H}), 5.41(\mathrm{dd}, J=8.3,8.3 \mathrm{~Hz}, 1 \mathrm{H}), 2.22-2.12$ $(\mathrm{m}, 2 \mathrm{H}), 2.14(\mathrm{~s}, 3 \mathrm{H}), 2.08(\mathrm{~s}, 3 \mathrm{H}), 2.02-1.91(\mathrm{~m}, 1 \mathrm{H}), 1.84-1.74(\mathrm{~m}$, 2H), 1.81 (d, $J=7.2 \mathrm{~Hz}, 3 \mathrm{H}), 1.22$ (dd, $J=5.3,5.3 \mathrm{~Hz}, 1 \mathrm{H}), 1.06$ $(\mathrm{dd}, J=8.3,5.3 \mathrm{~Hz}, 1 \mathrm{H}) ;{ }^{13} \mathbf{C}$ NMR (75 $\left.\mathbf{~ M H z}, \mathbf{C D C l}_{3}\right) \delta 171.22$, $169.29,131.10,123.20,97.00,79.39,71.03,28.36,26.43,24.83$,

21.79, 21.33, 21.06, 15.36, 13.54; IR (neat): $v_{\max }=2921,2219,1761,1738,1237 \mathrm{~cm}^{-1} ; \mathbf{H R M S ~} \mathbf{m} / \mathbf{z}$ (ESI): calc. for $\mathrm{C}_{15} \mathrm{H}_{18} \mathrm{O}_{4} \mathrm{Na}\left[\mathrm{M}^{+}+\mathrm{Na}\right] 285.1103$, found 285.1109 . 


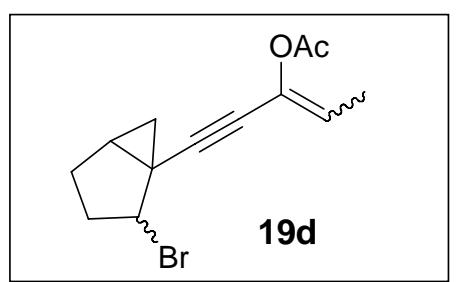

Colorless oil (50\% yield): ${ }^{1} \mathbf{H}$ NMR (300 MHz, $\left.\mathbf{C D C l}_{3}\right) \delta 5.68$ (q, $J$ $=7.2 \mathrm{~Hz}, 1 \mathrm{H}), 4.55(\mathrm{dd}, J=9.7,8.0 \mathrm{~Hz}, 1 \mathrm{H}), 2.32-2.19(\mathrm{~m}, 2 \mathrm{H})$, 2.14 (s, 3H), 2.03-1.95 (m, 2H), 1.85 (d, J=7.2 Hz, 3H), 1.69-1.62 (m, 1H), 1.18-1.08 (m, 2H); ${ }^{13}$ C NMR (75 MHz, $\mathbf{C D C l}_{3}$ ) $\delta$ 169.31, $131.01,123.72,96.09,72.04,55.77,32.50,32.45,26.73,24.91$, 21.07, 17.66, 13.69; IR (neat): $v_{\max }=2937,2212,1743,1218 \mathrm{~cm}^{-1}$; HRMS $\boldsymbol{m} / \mathbf{z}$ (ESI): calc. for $\mathrm{C}_{13} \mathrm{H}_{15} \mathrm{BrO}_{2} \mathrm{Na}\left[\mathrm{M}^{+}+\mathrm{Na}\right] 305.0153$, found 305.0158. 


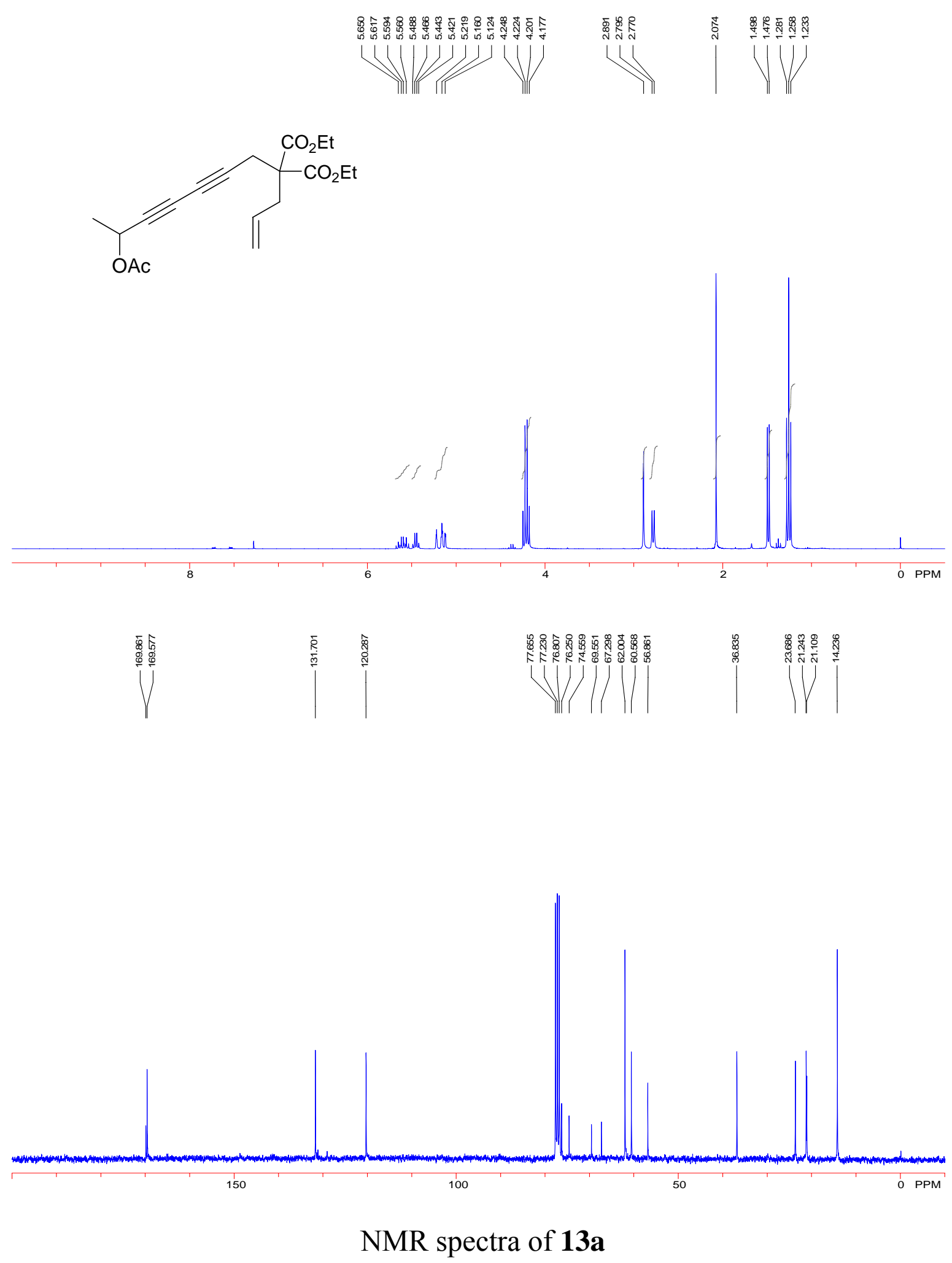




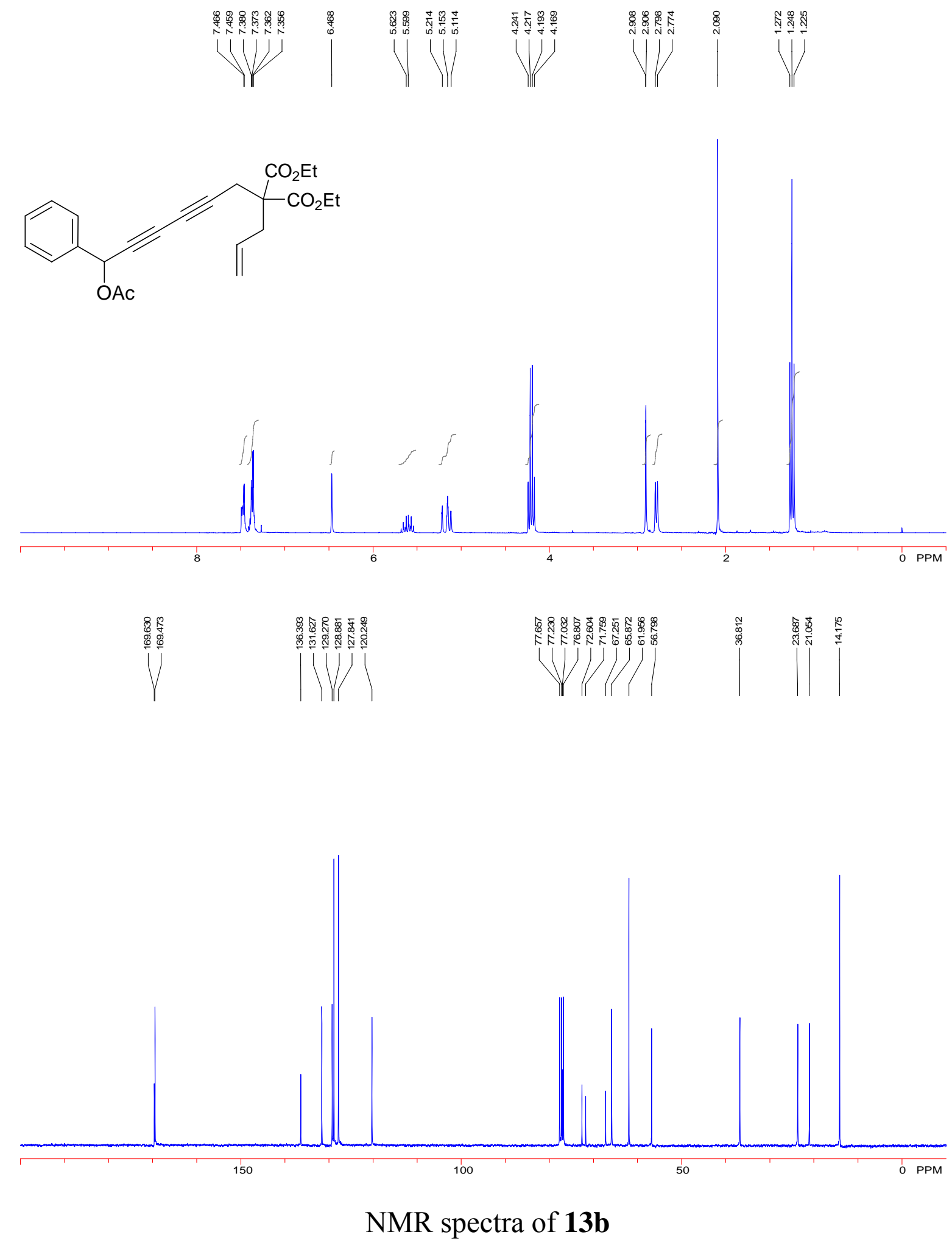




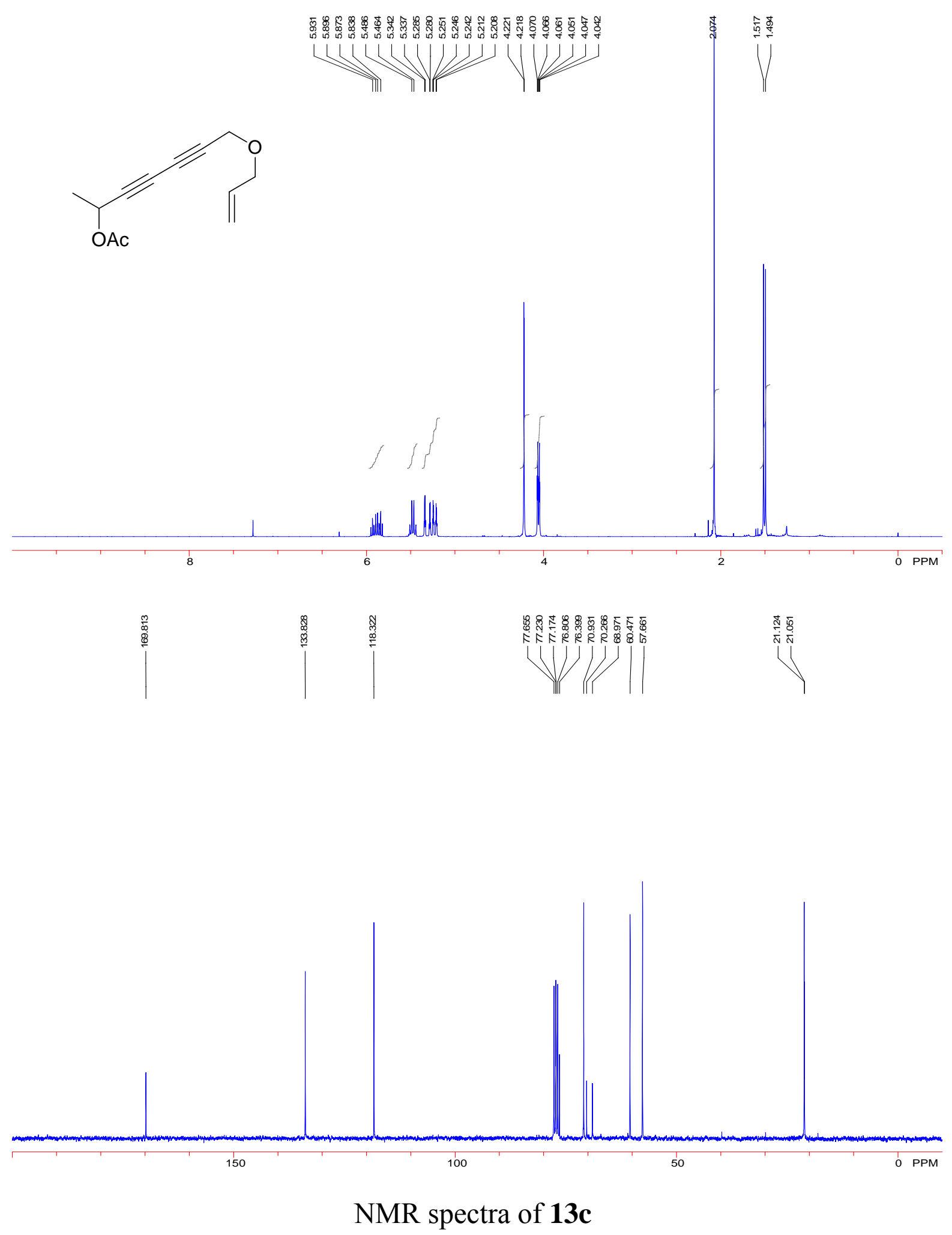




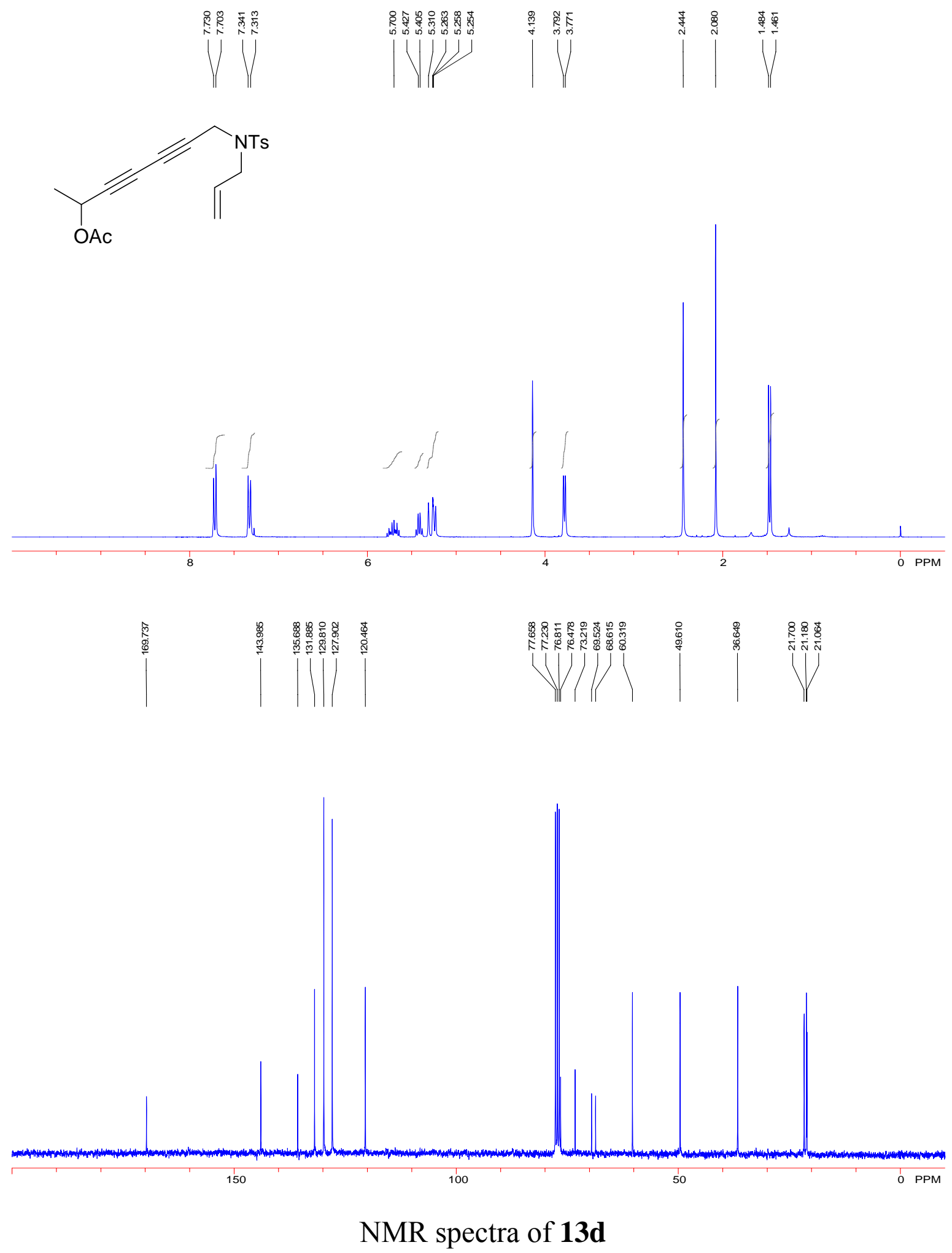




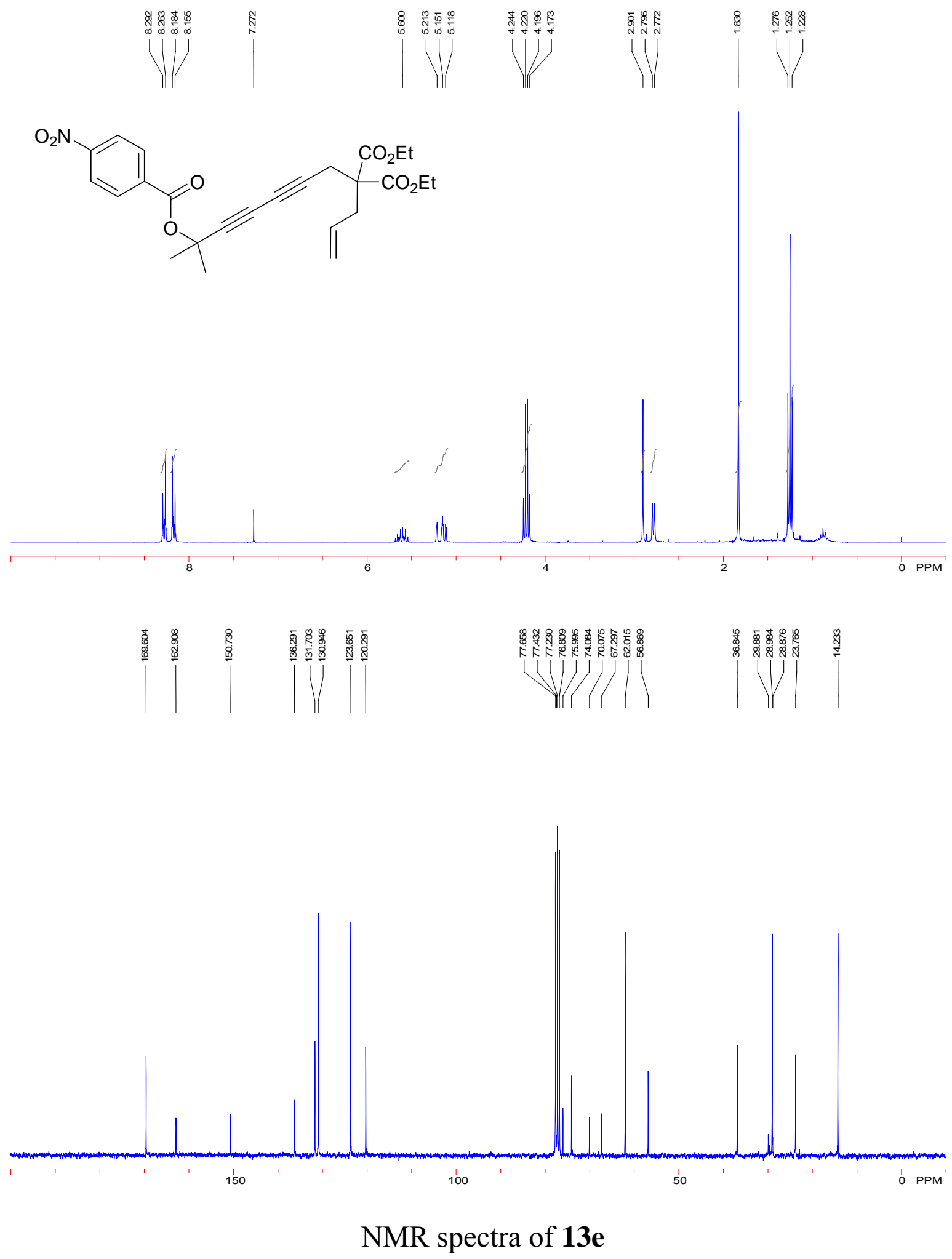




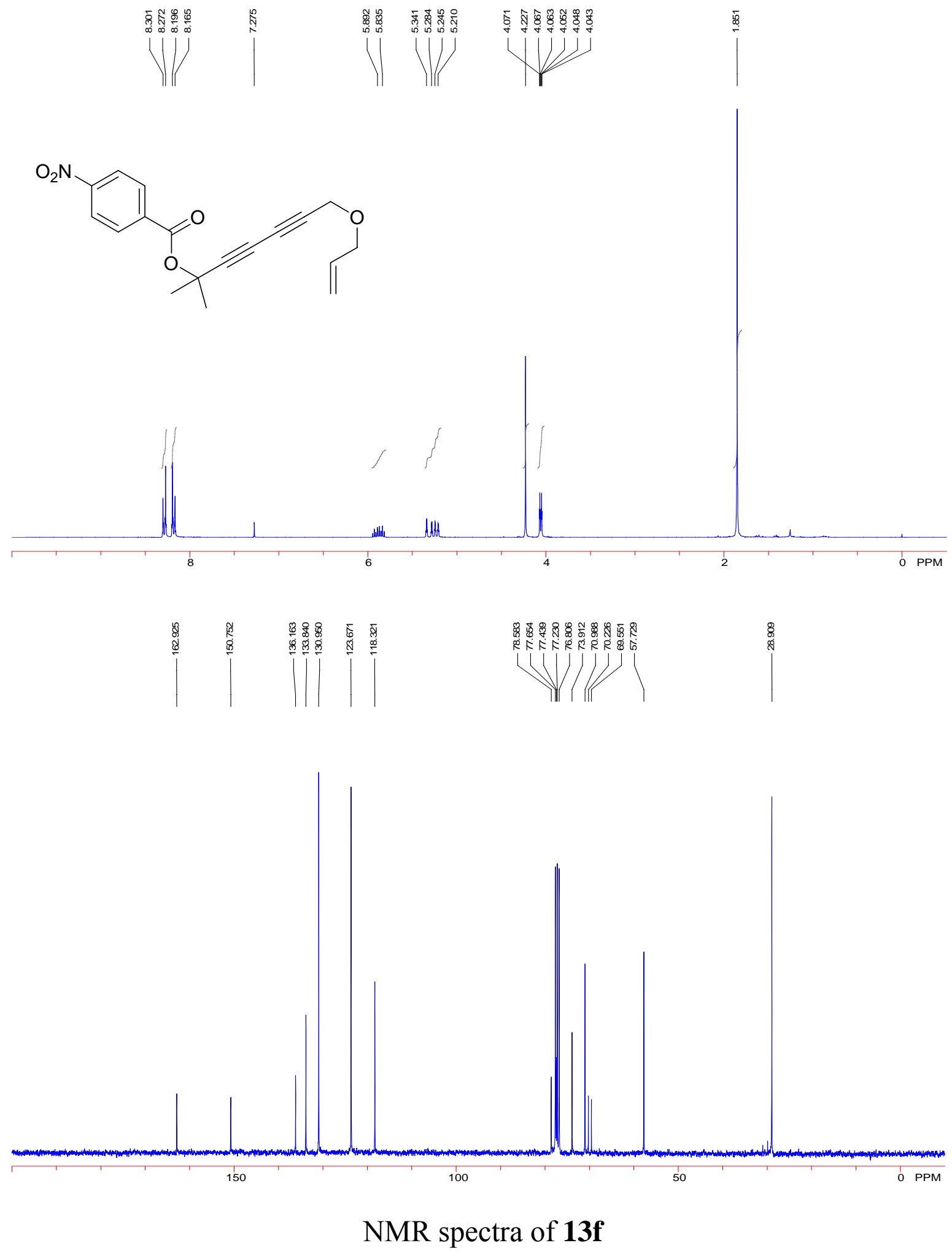




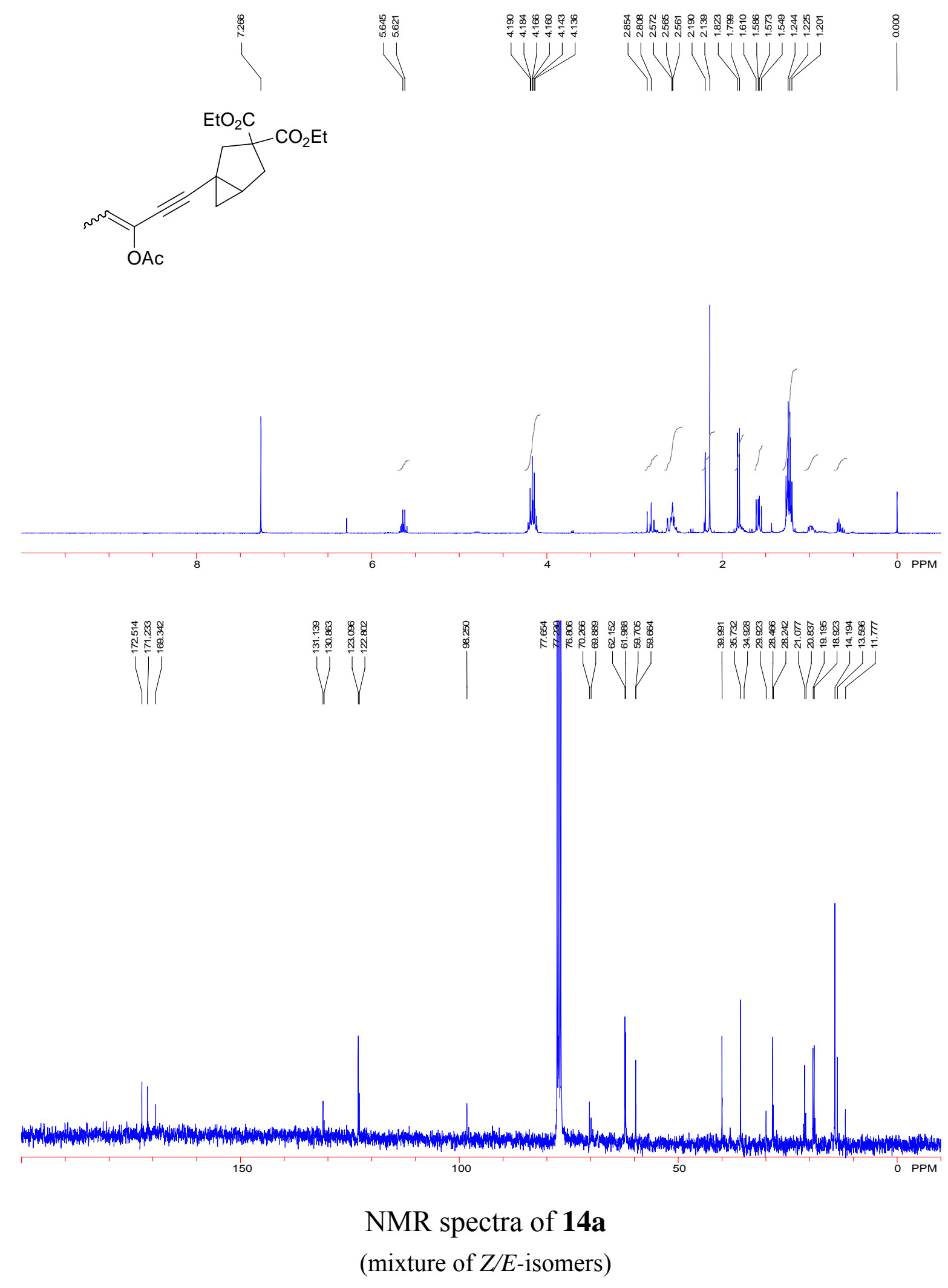



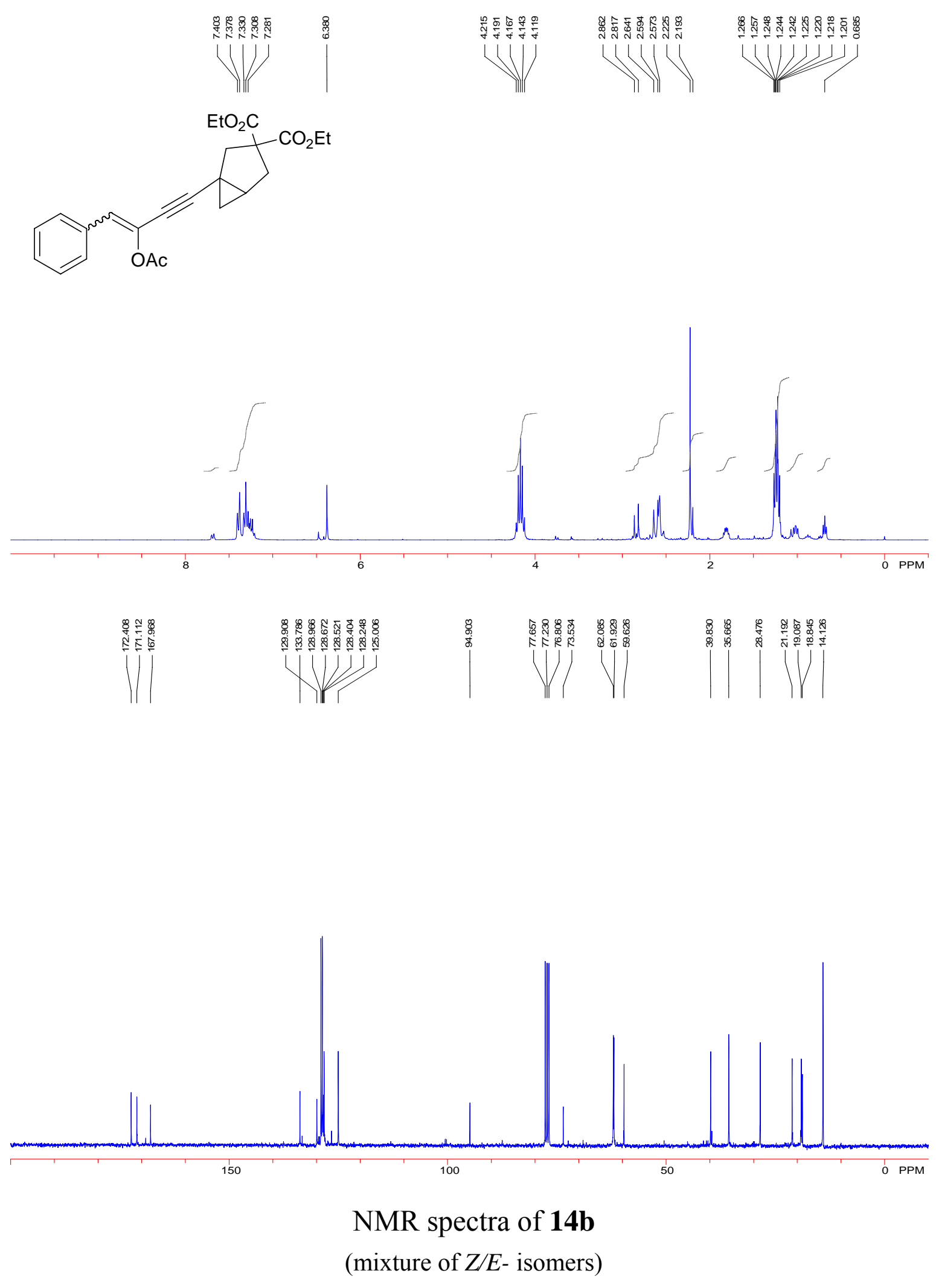


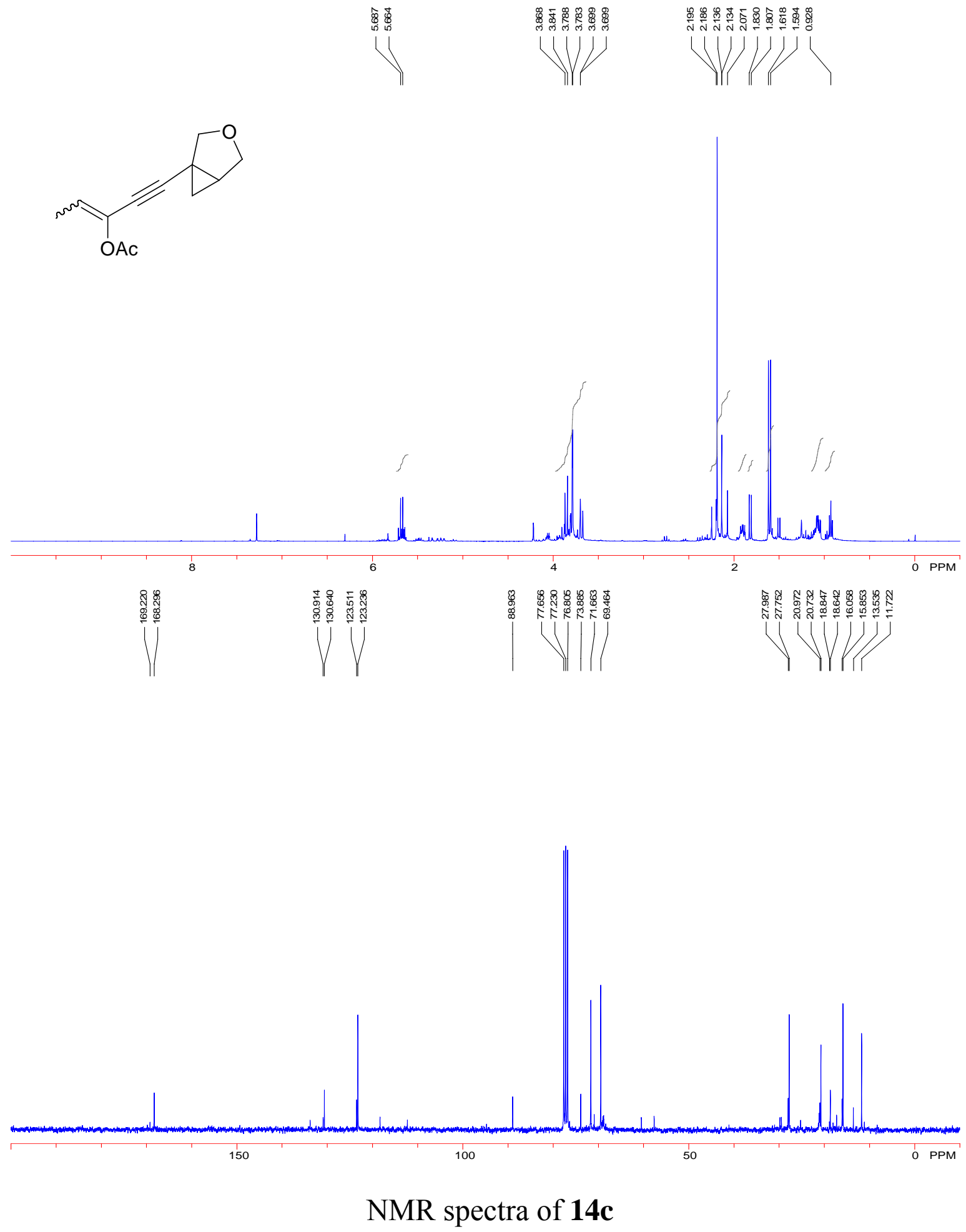

(mixture of $Z / E$ - isomers- crude; Purification attempt has led to a change of $Z / E$ ratio and the hydrolysis of acetate) 


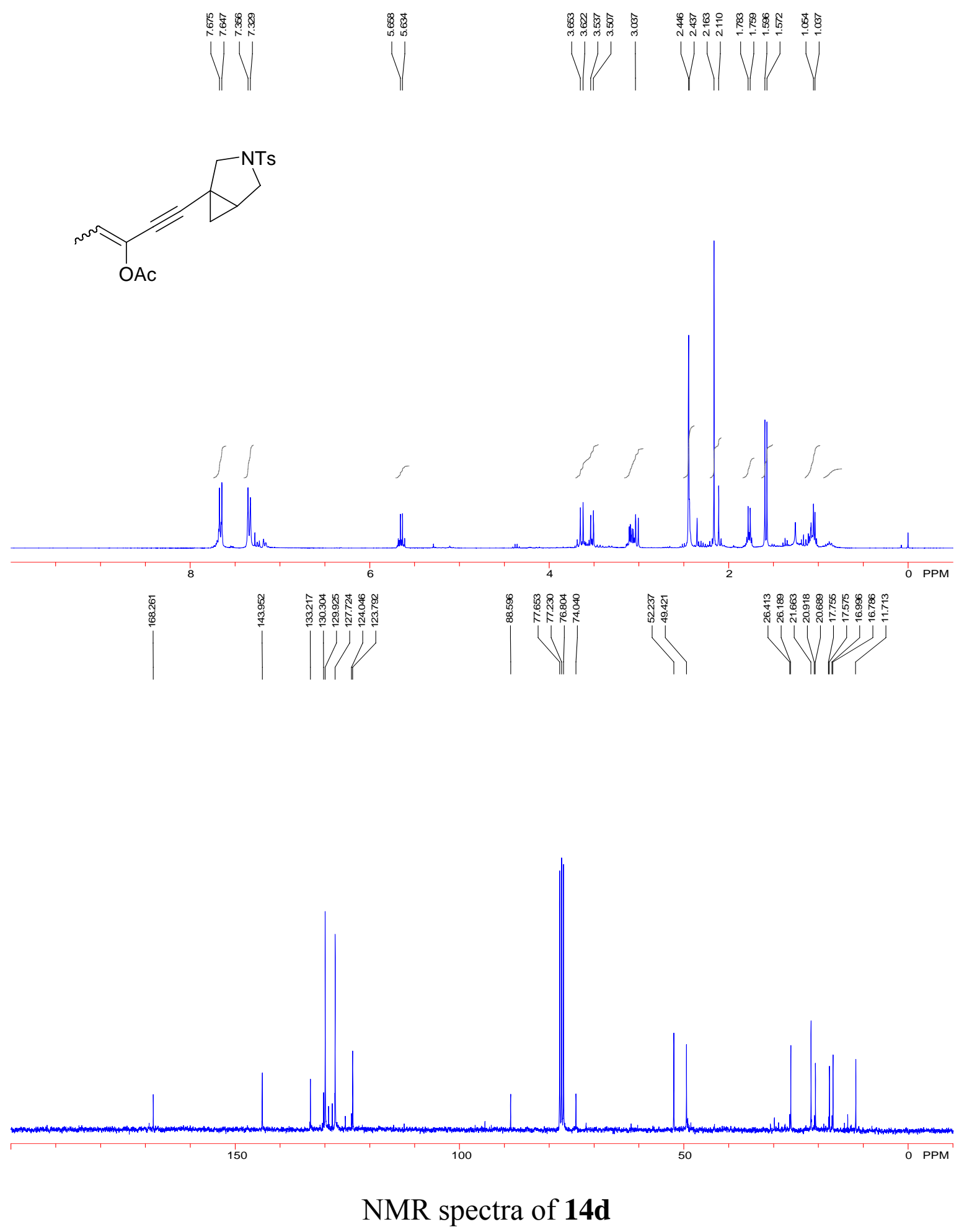

(mixture of $Z / E$ - isomers- crude; Purification attempt has led to a change of $Z / E$ ratio and the hydrolysis of acetate) 


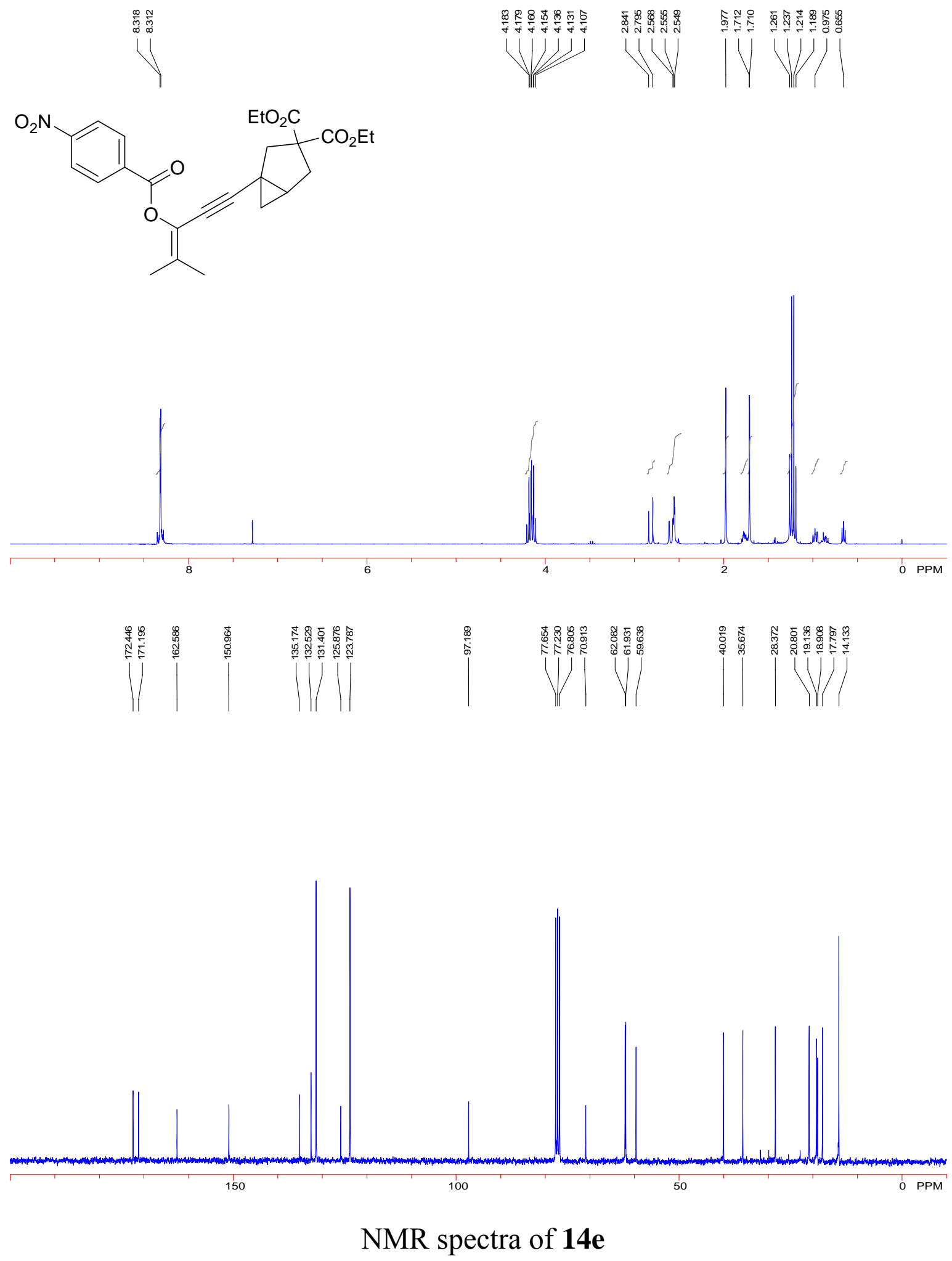



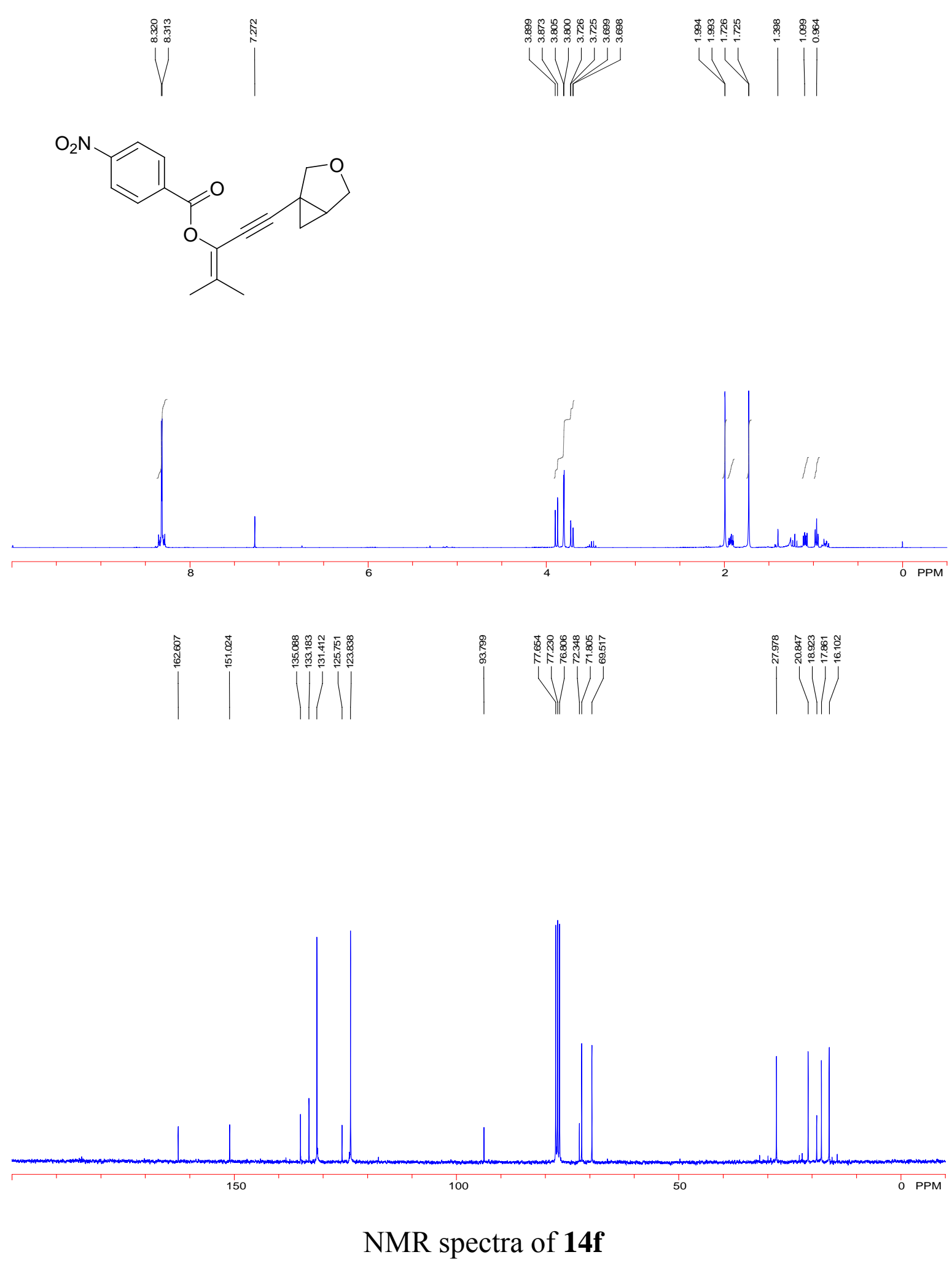


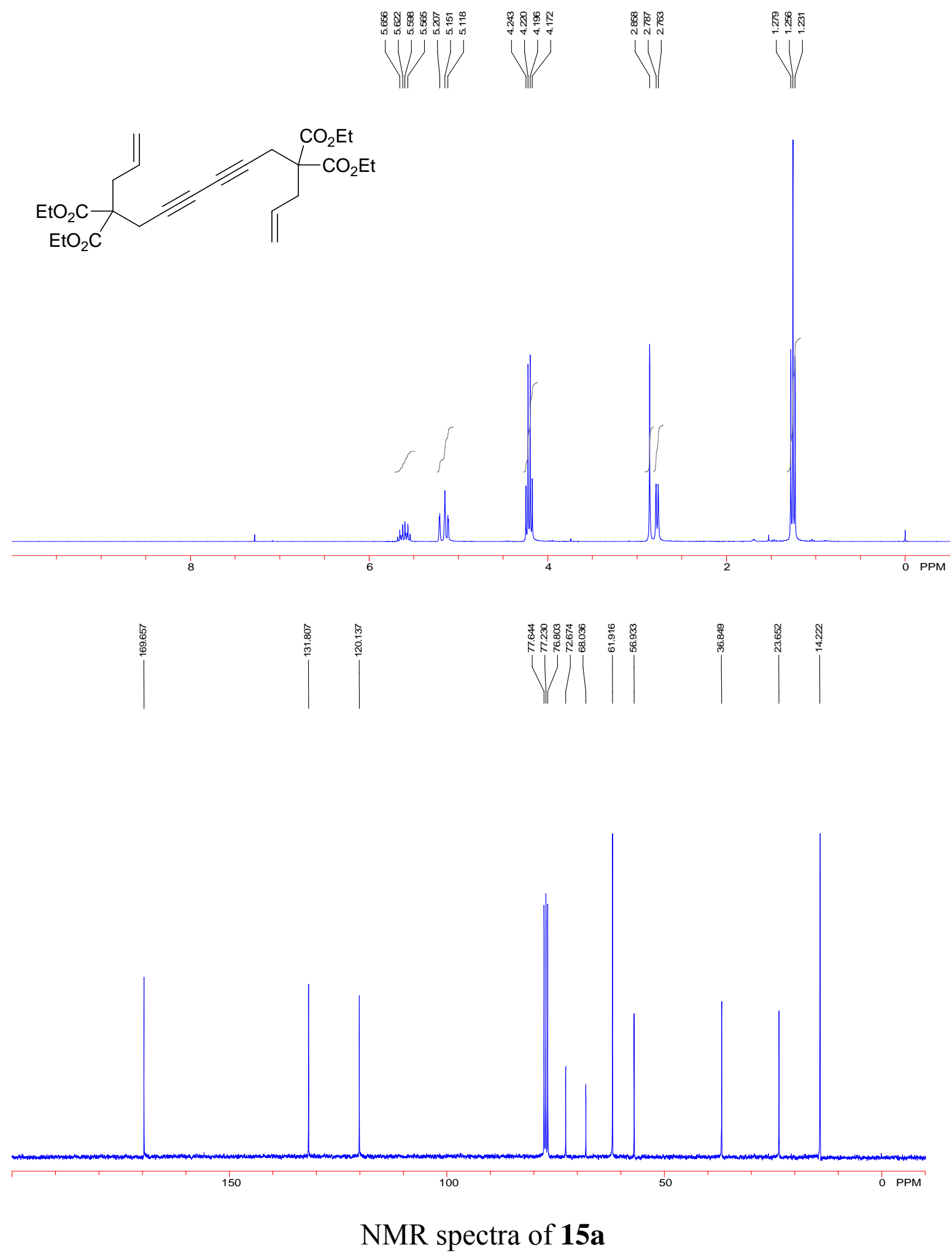




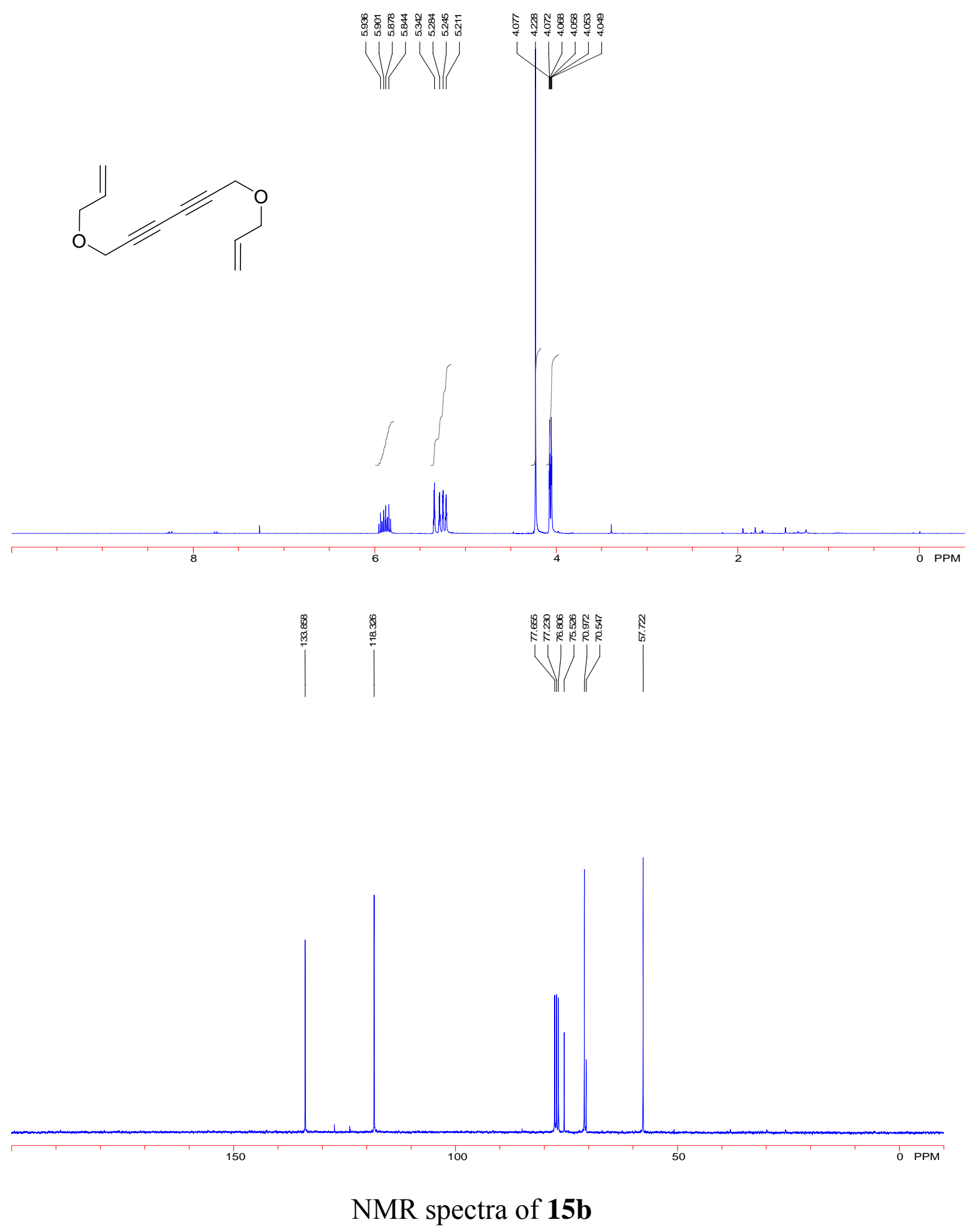




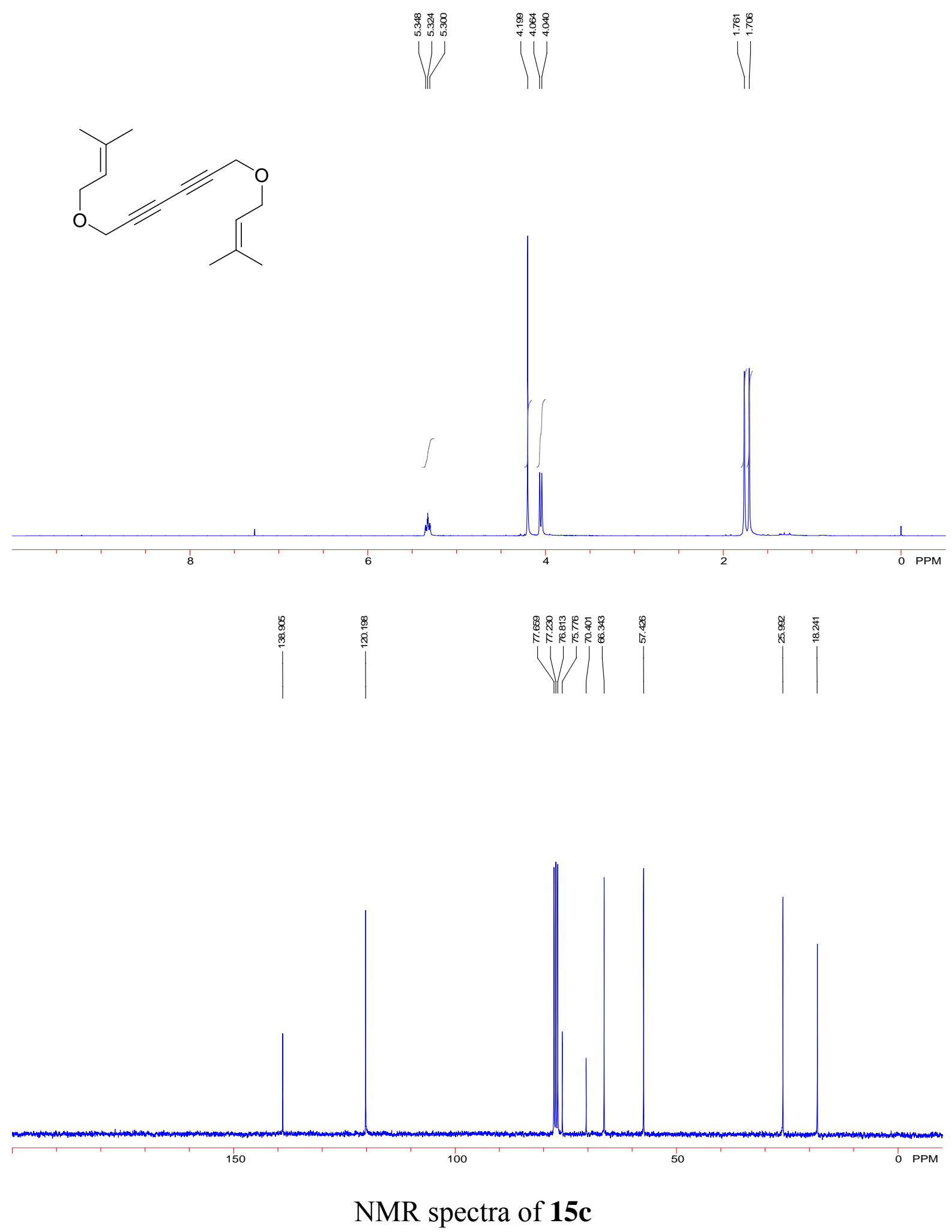




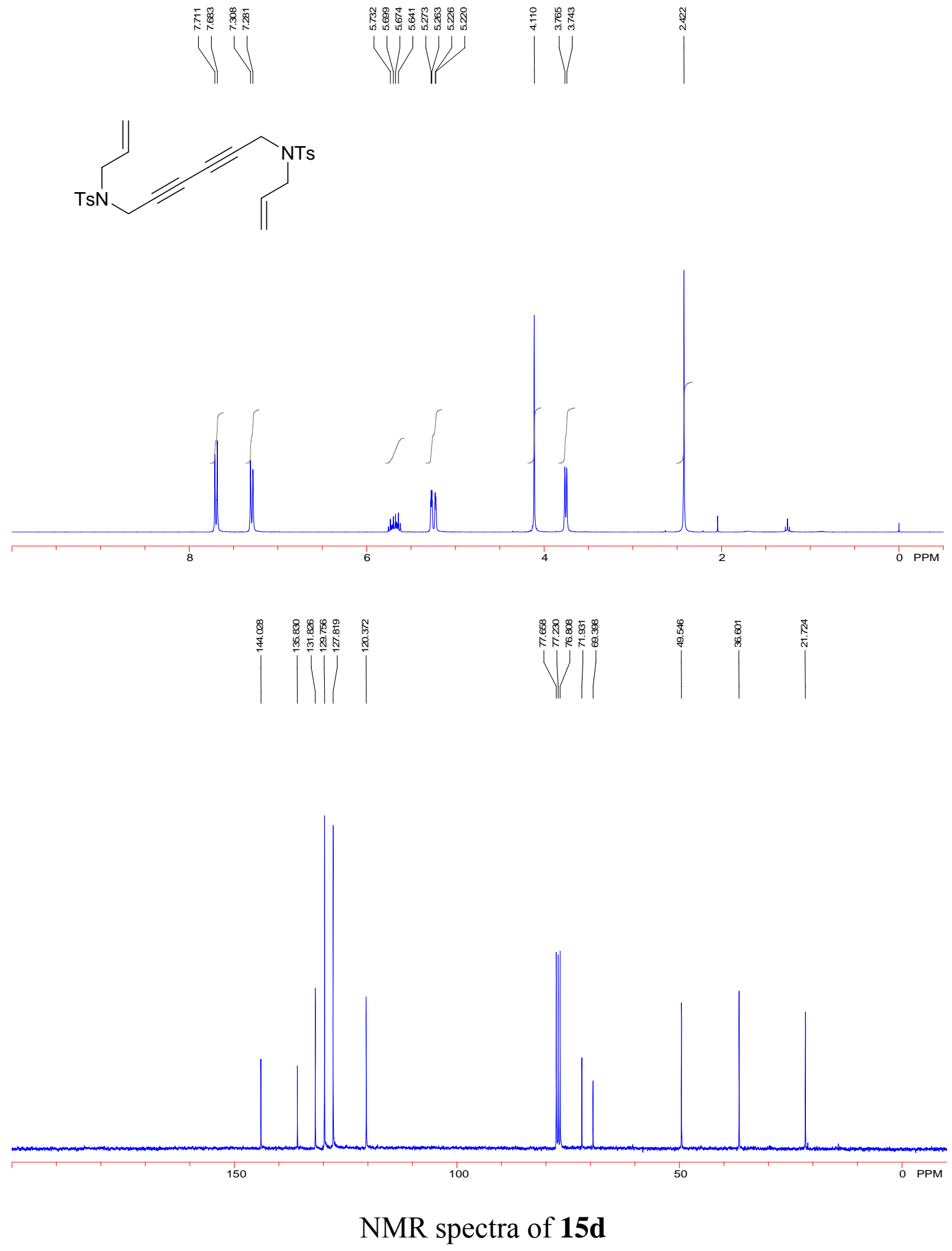



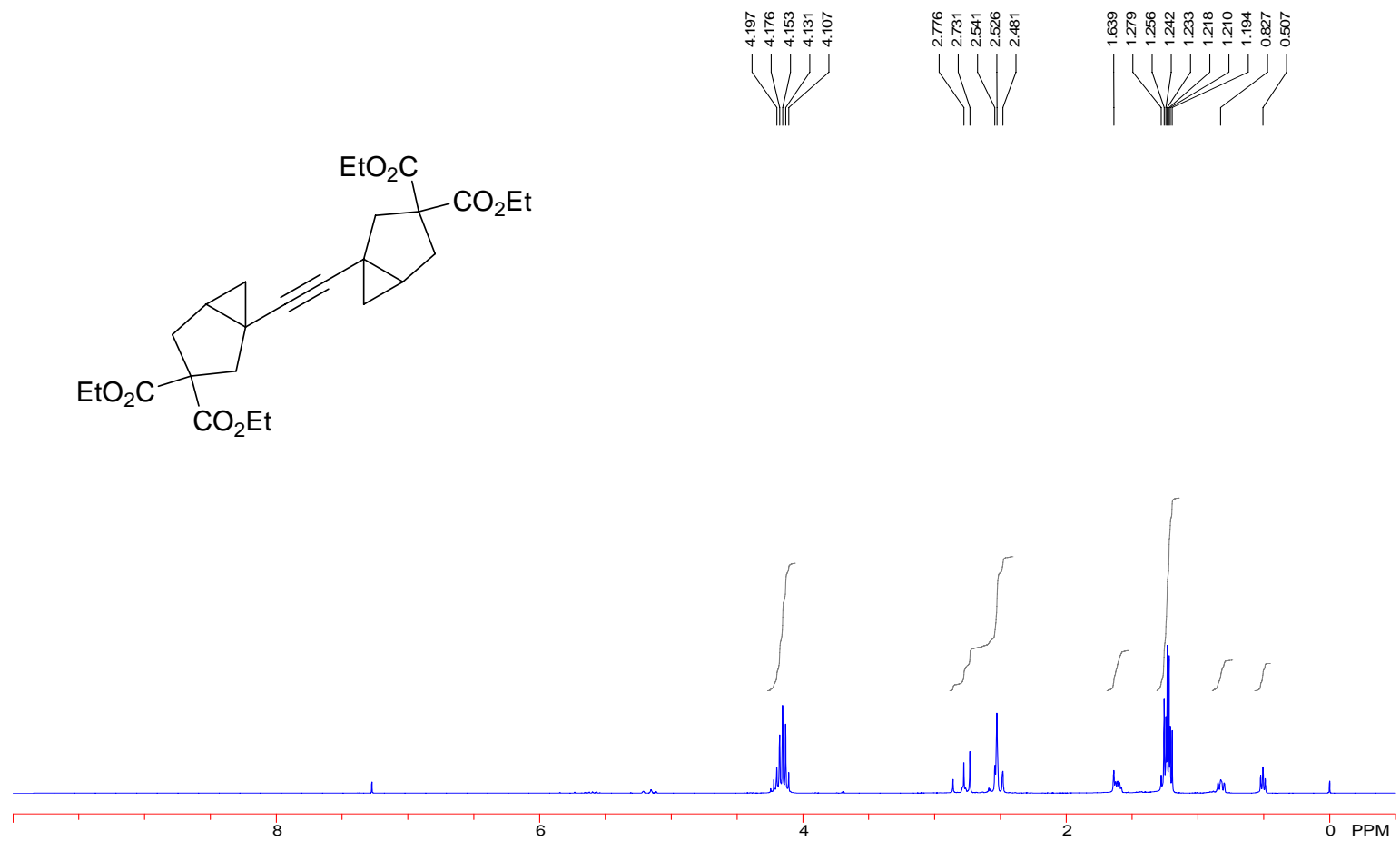

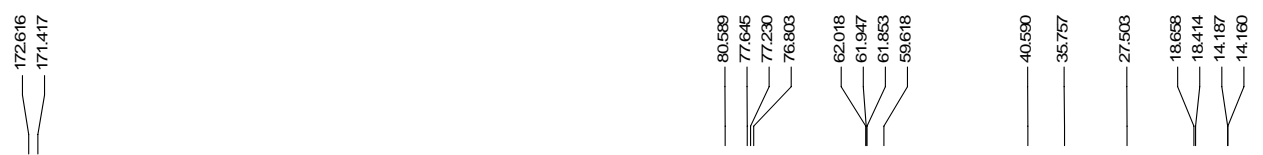

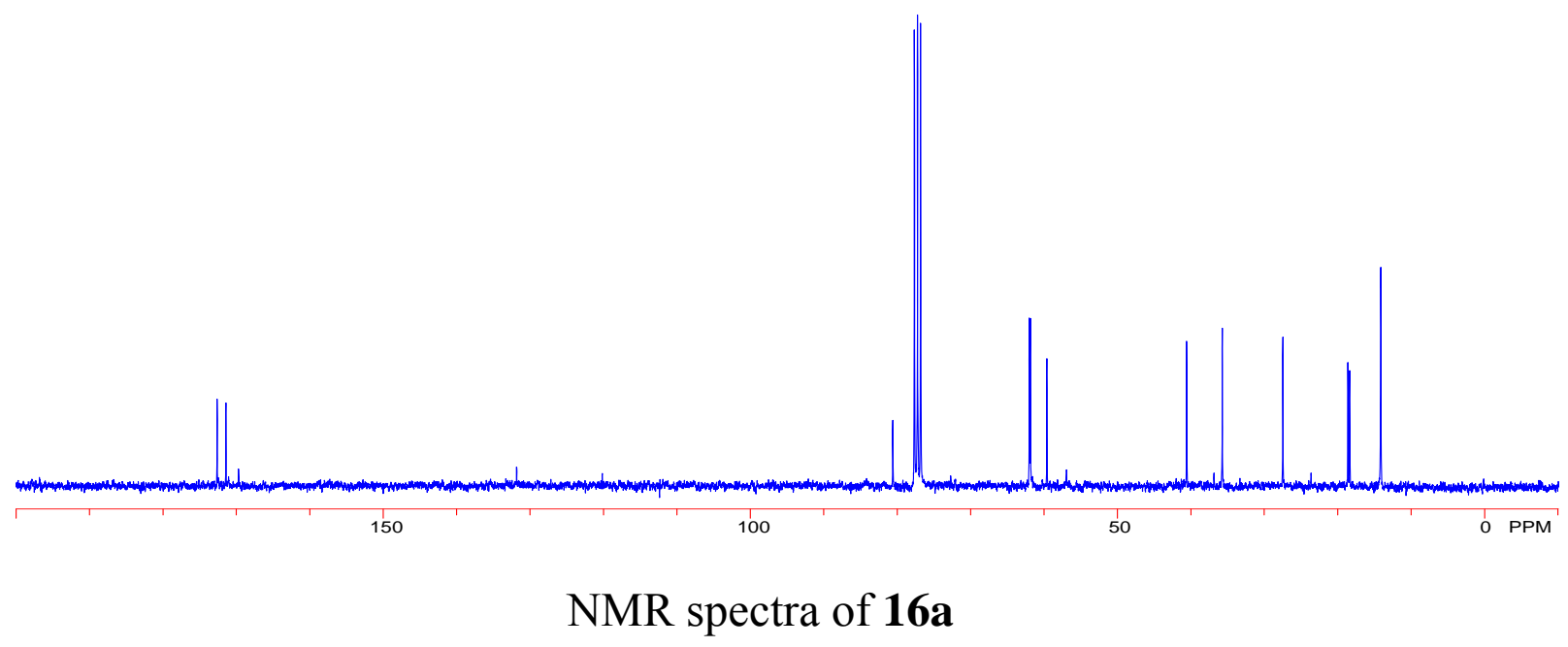




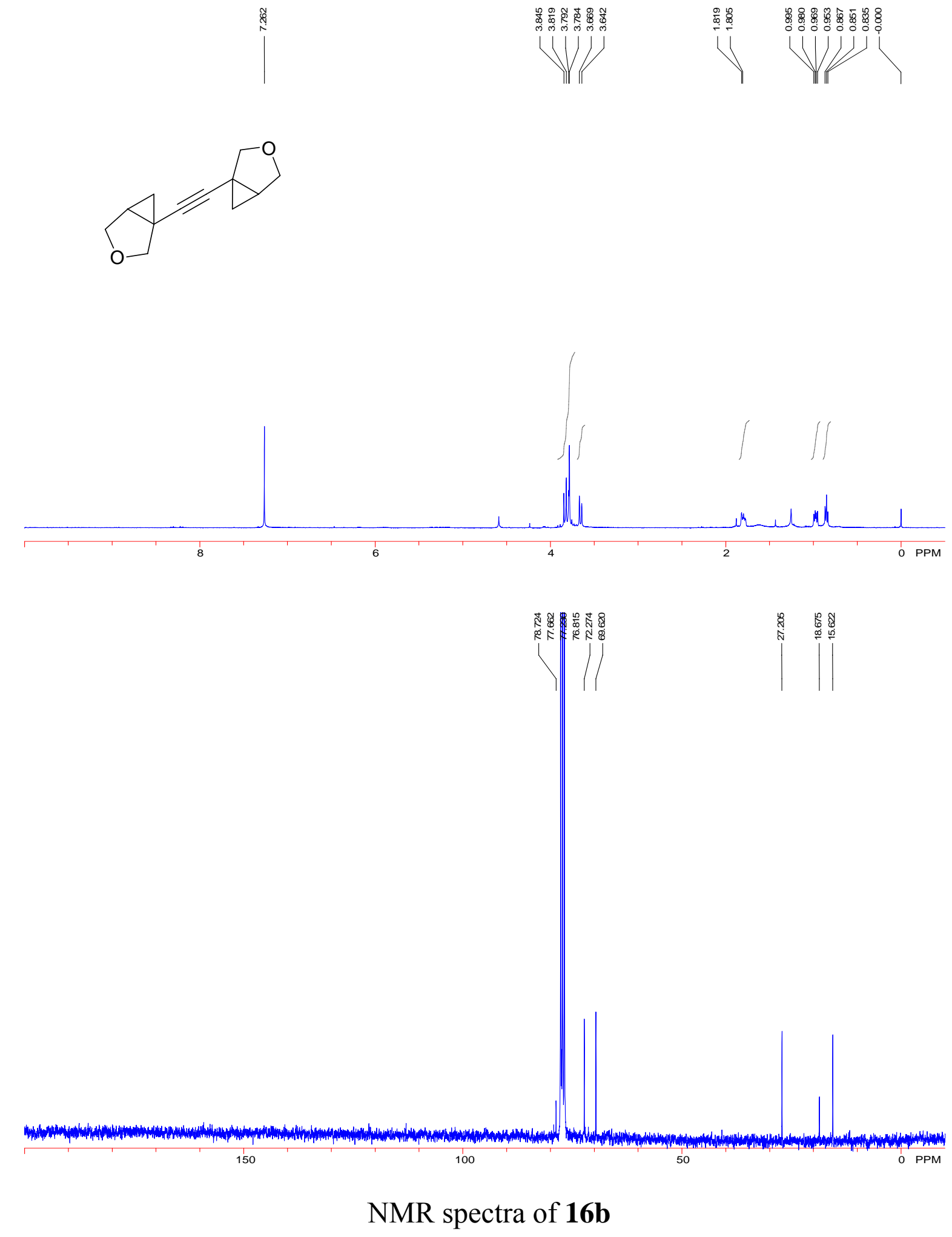




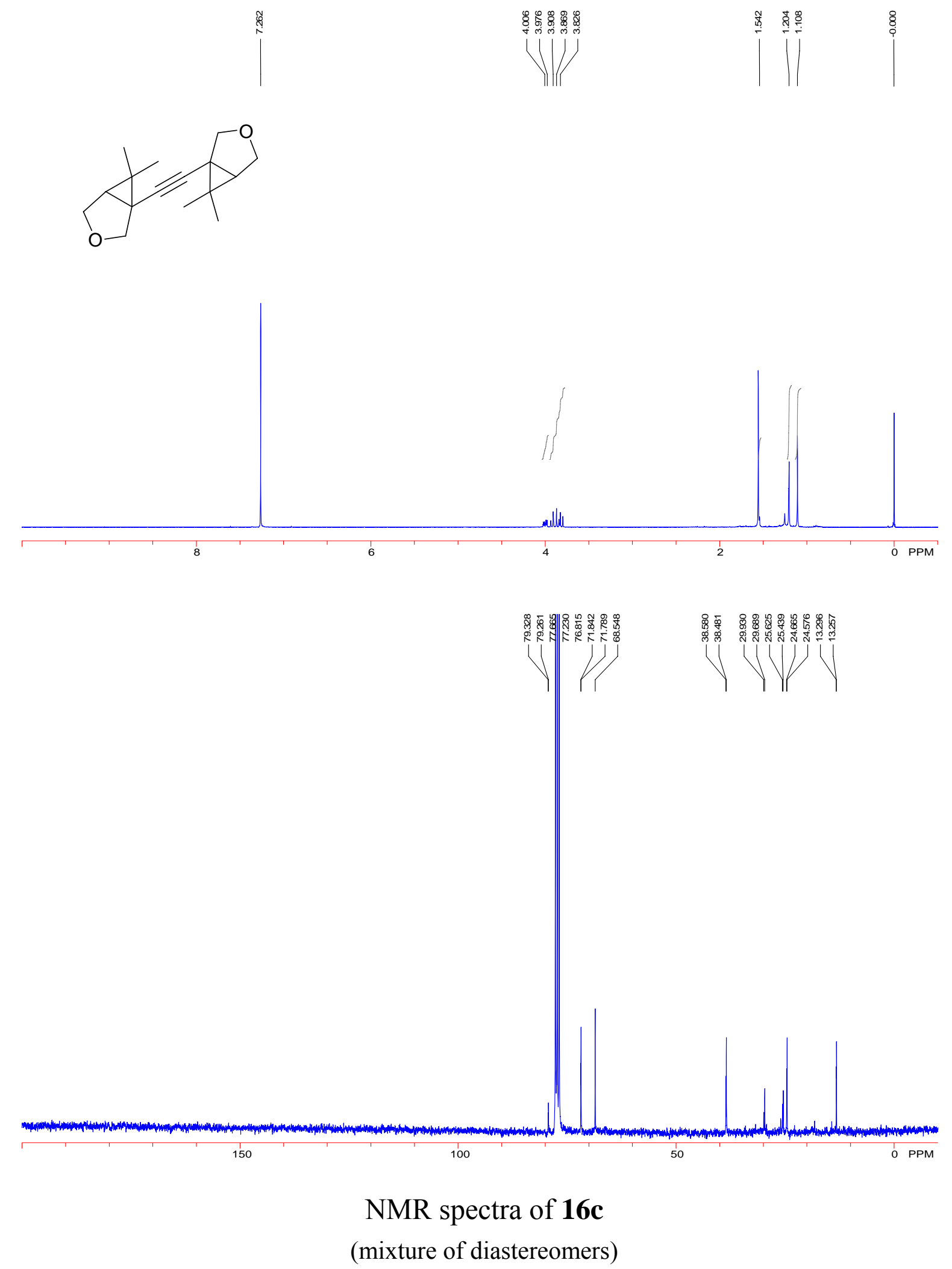



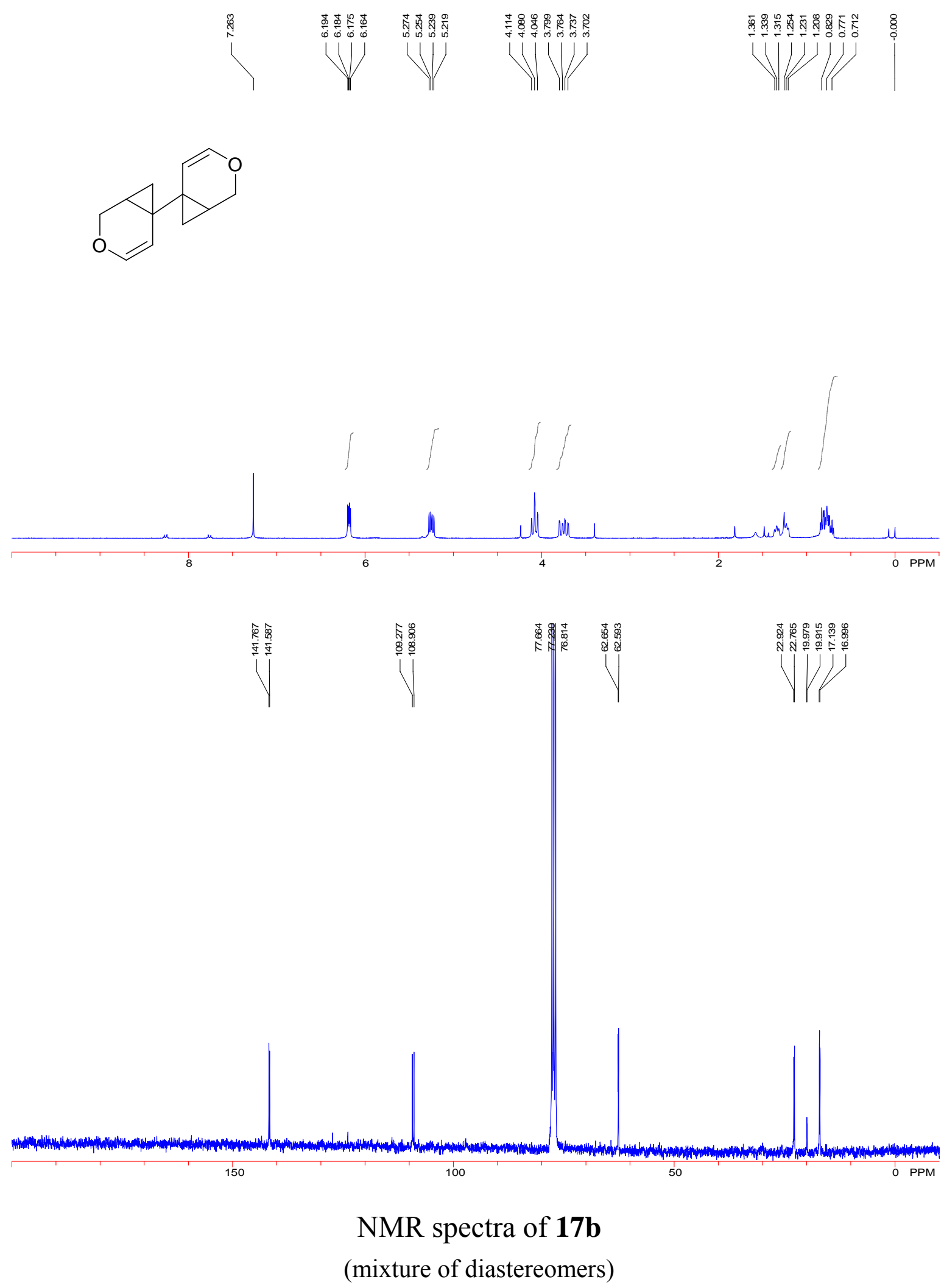

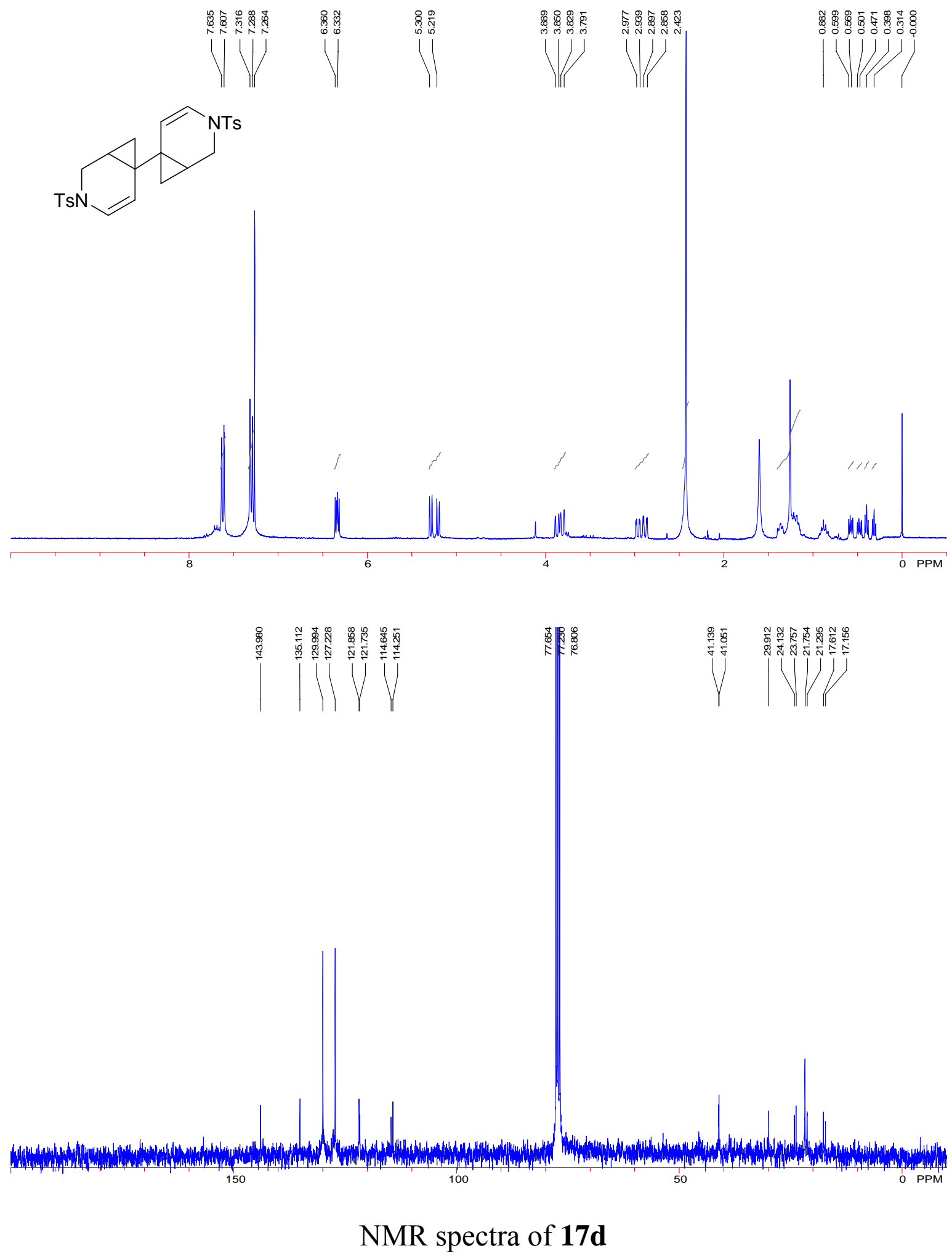

(mixture of diastereomers) 


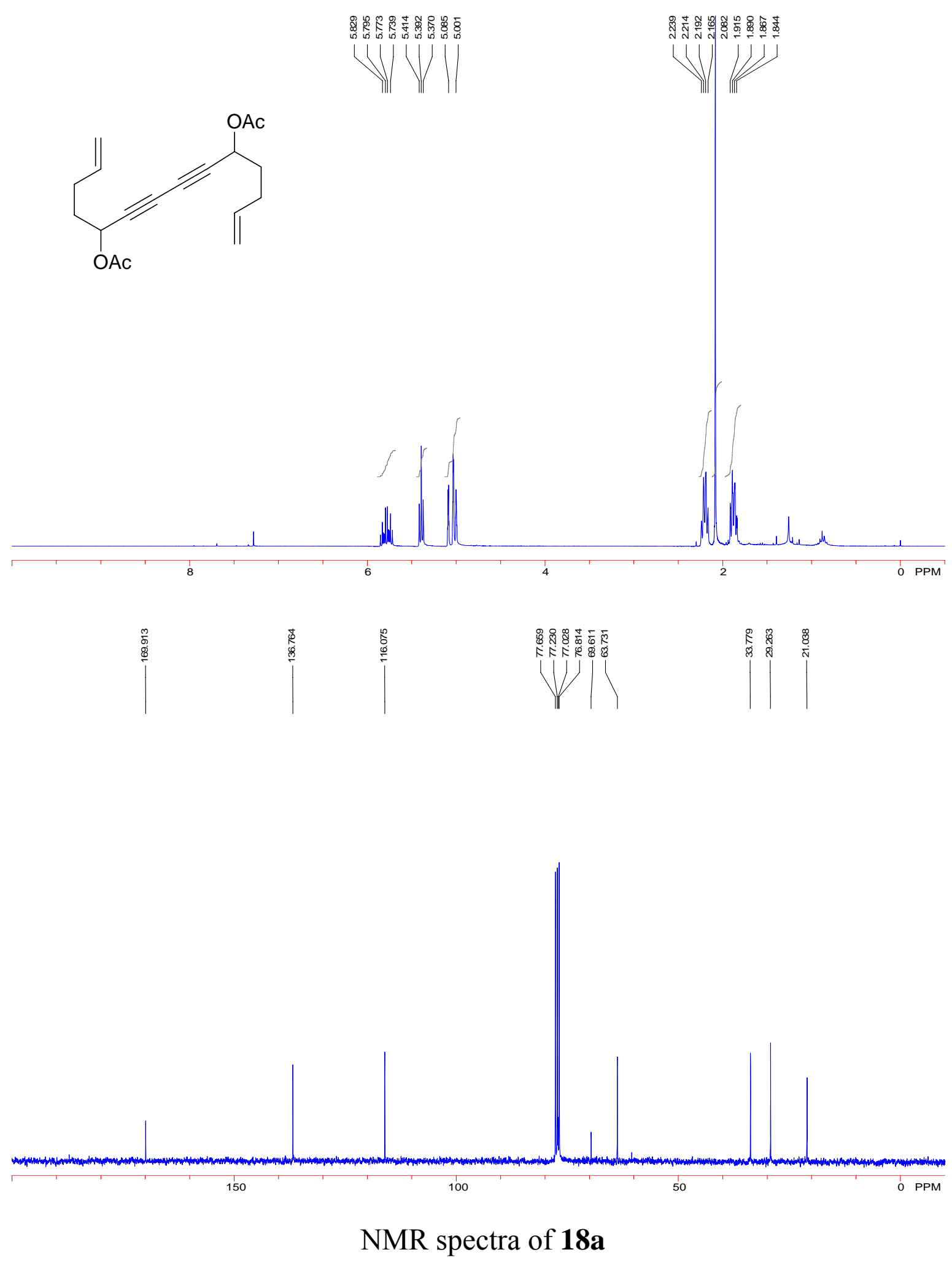



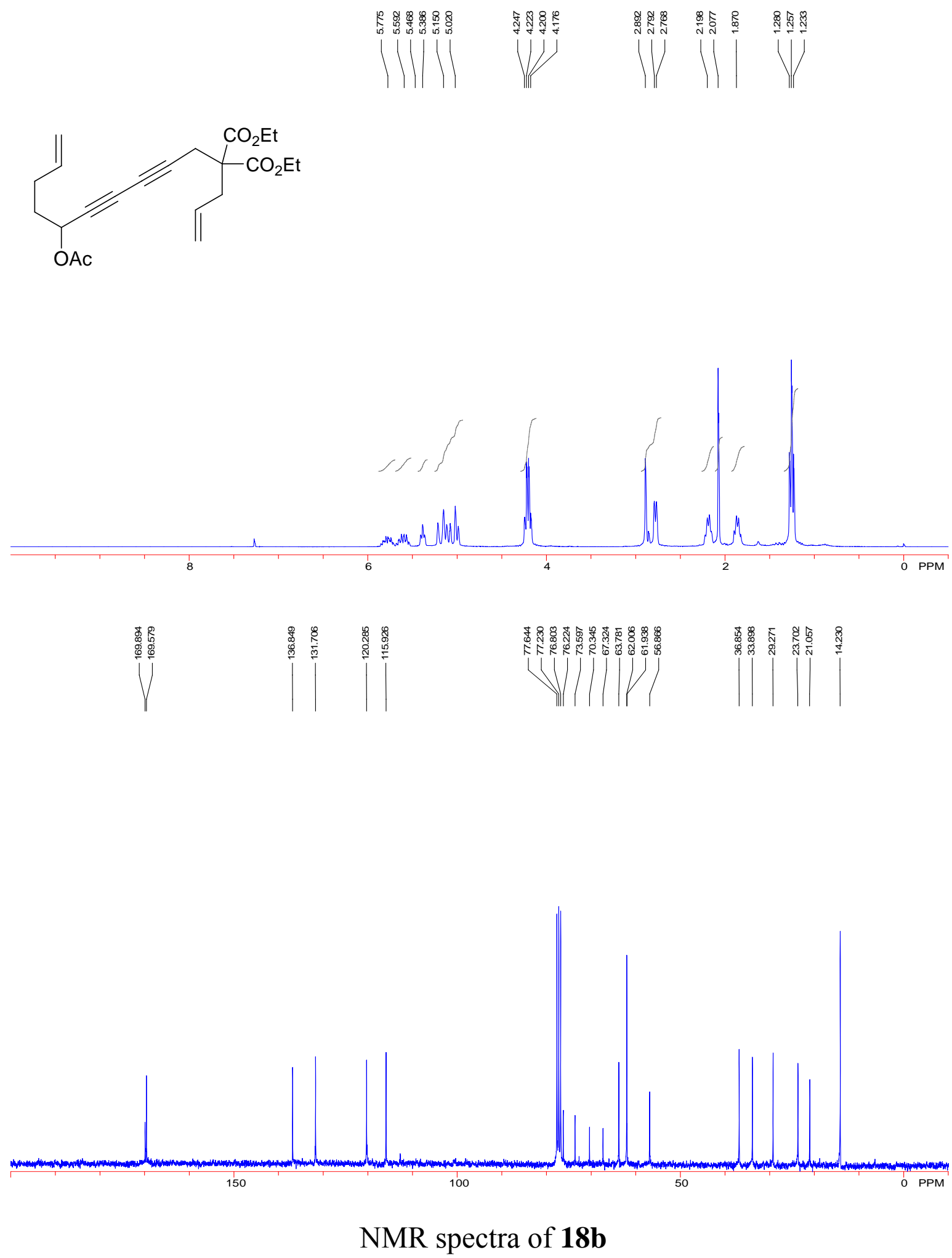


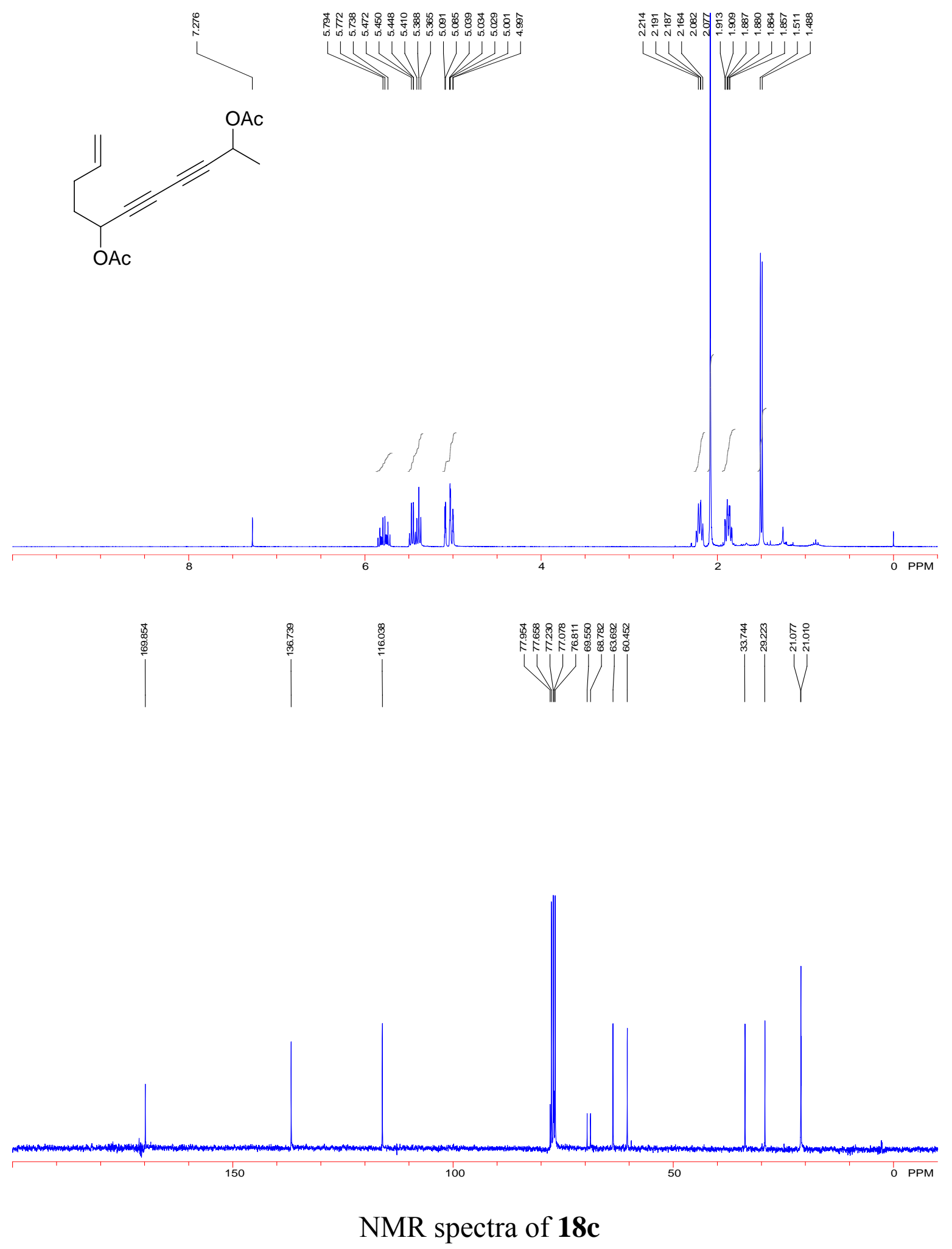




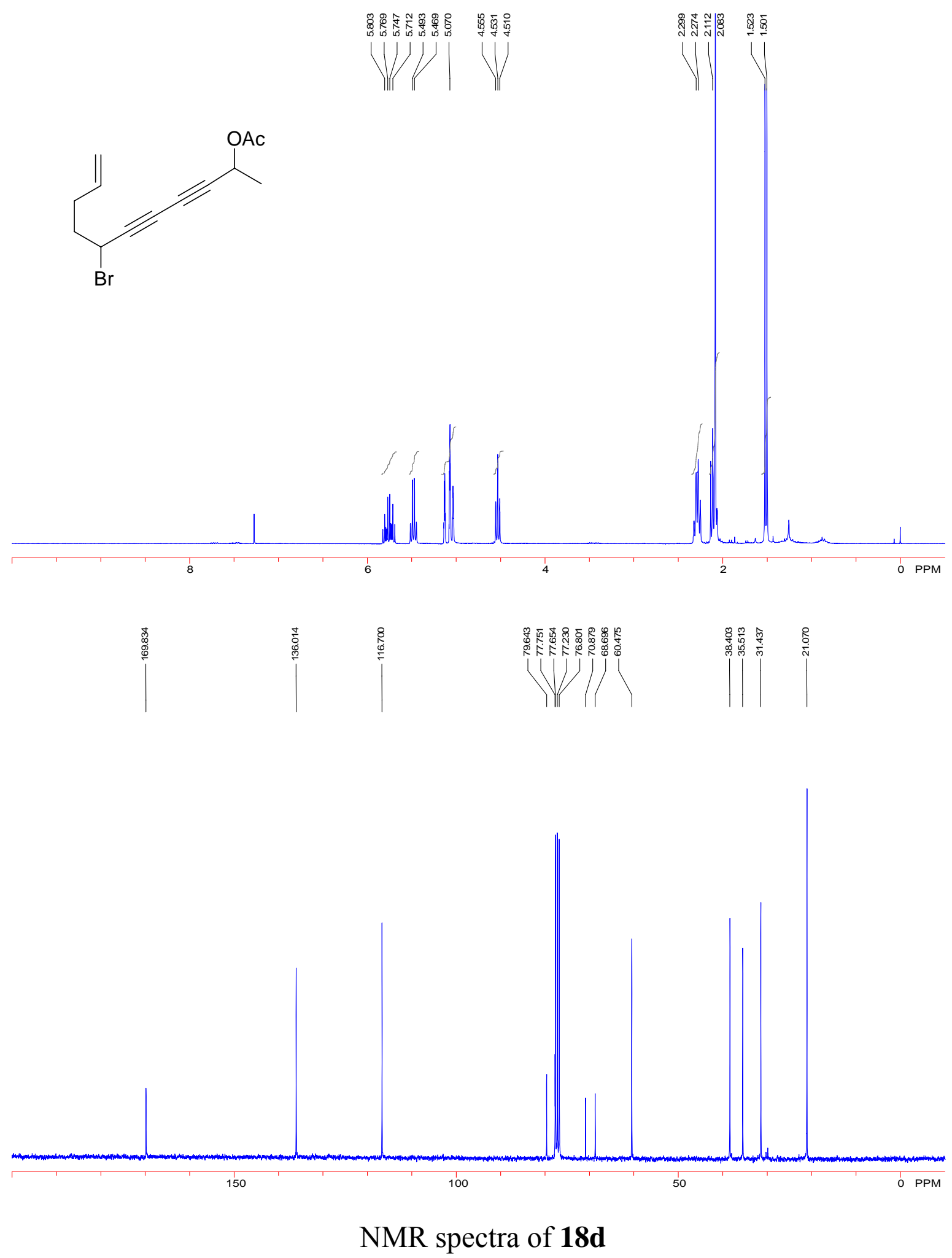




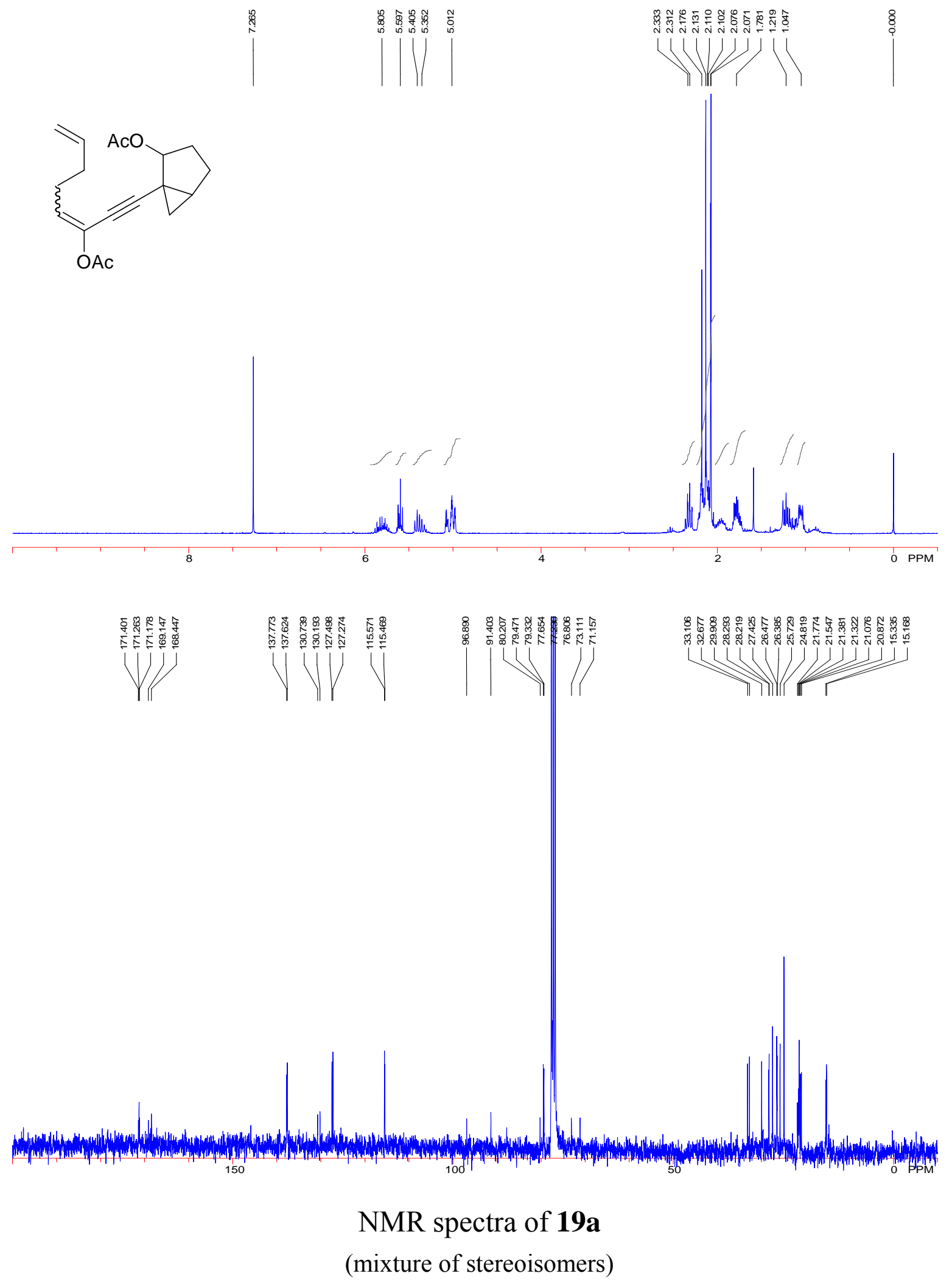



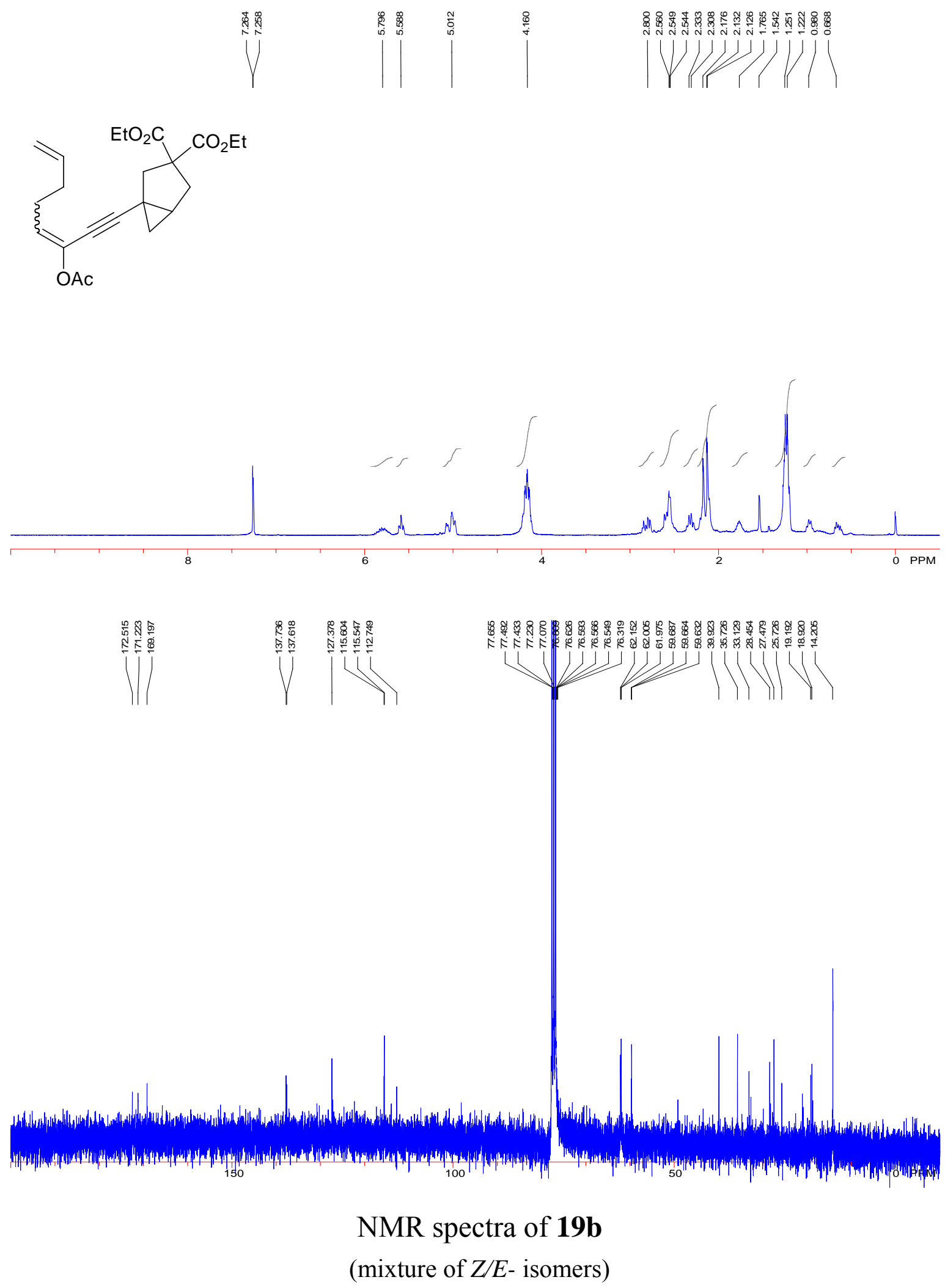


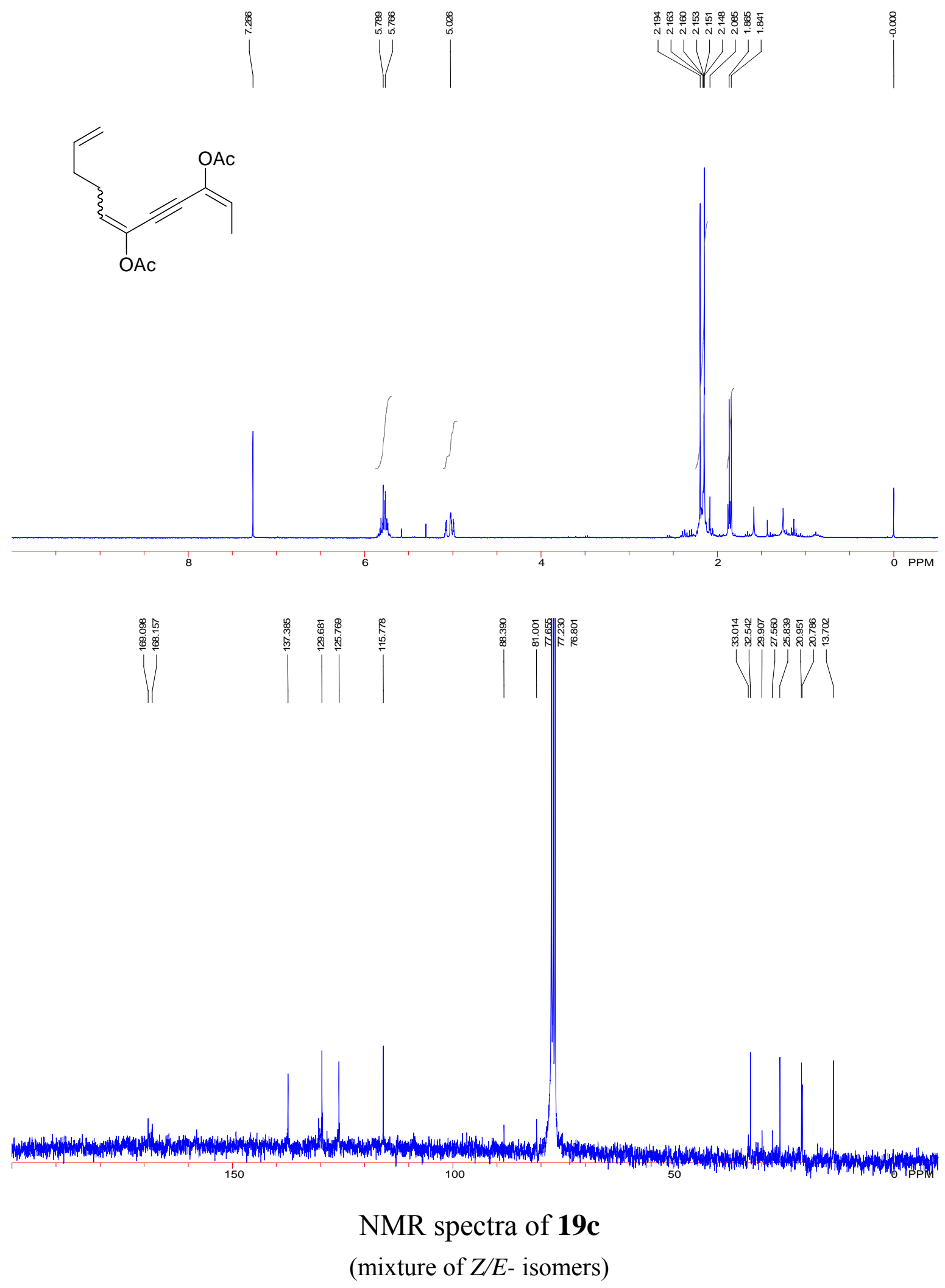




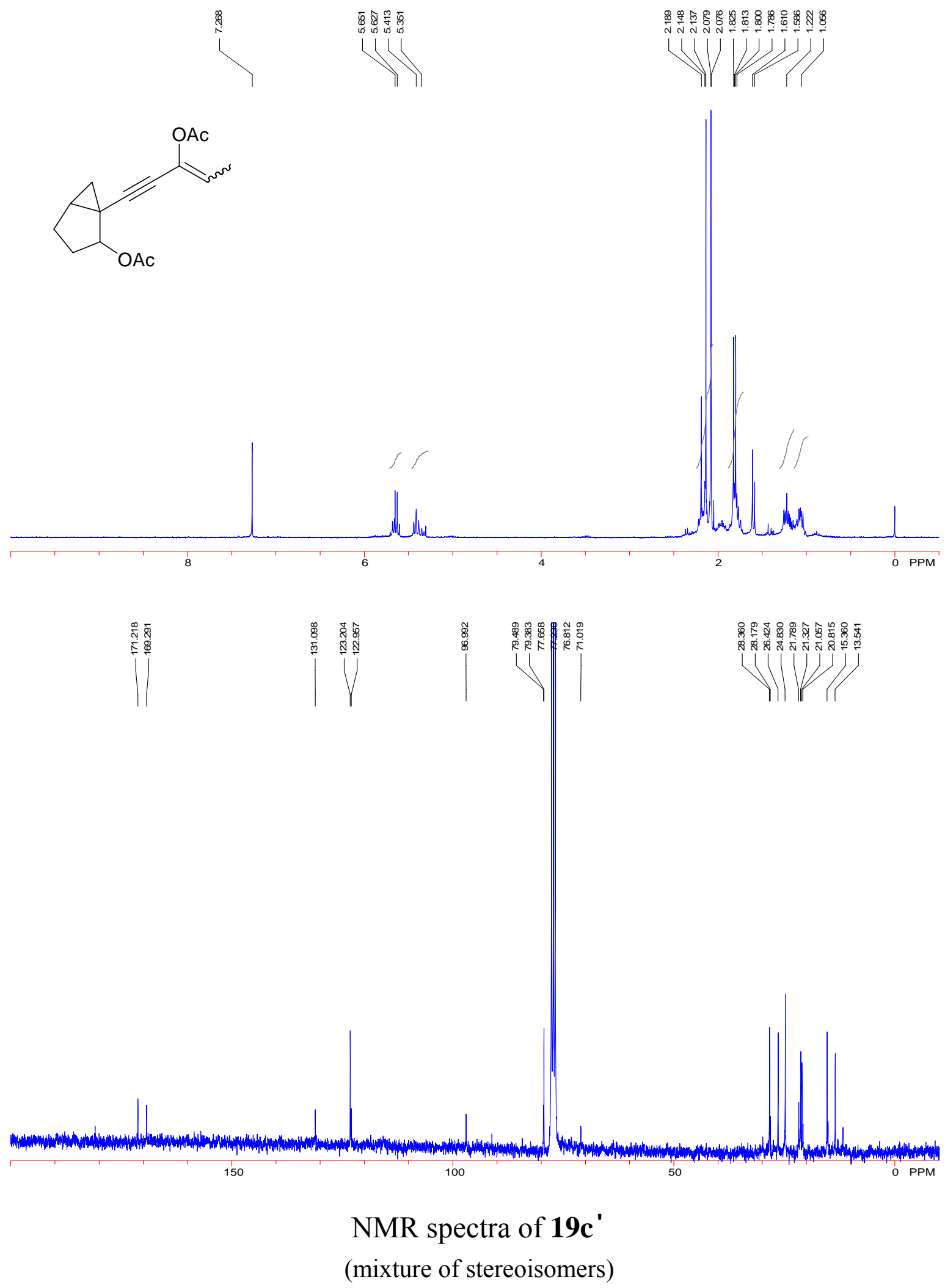




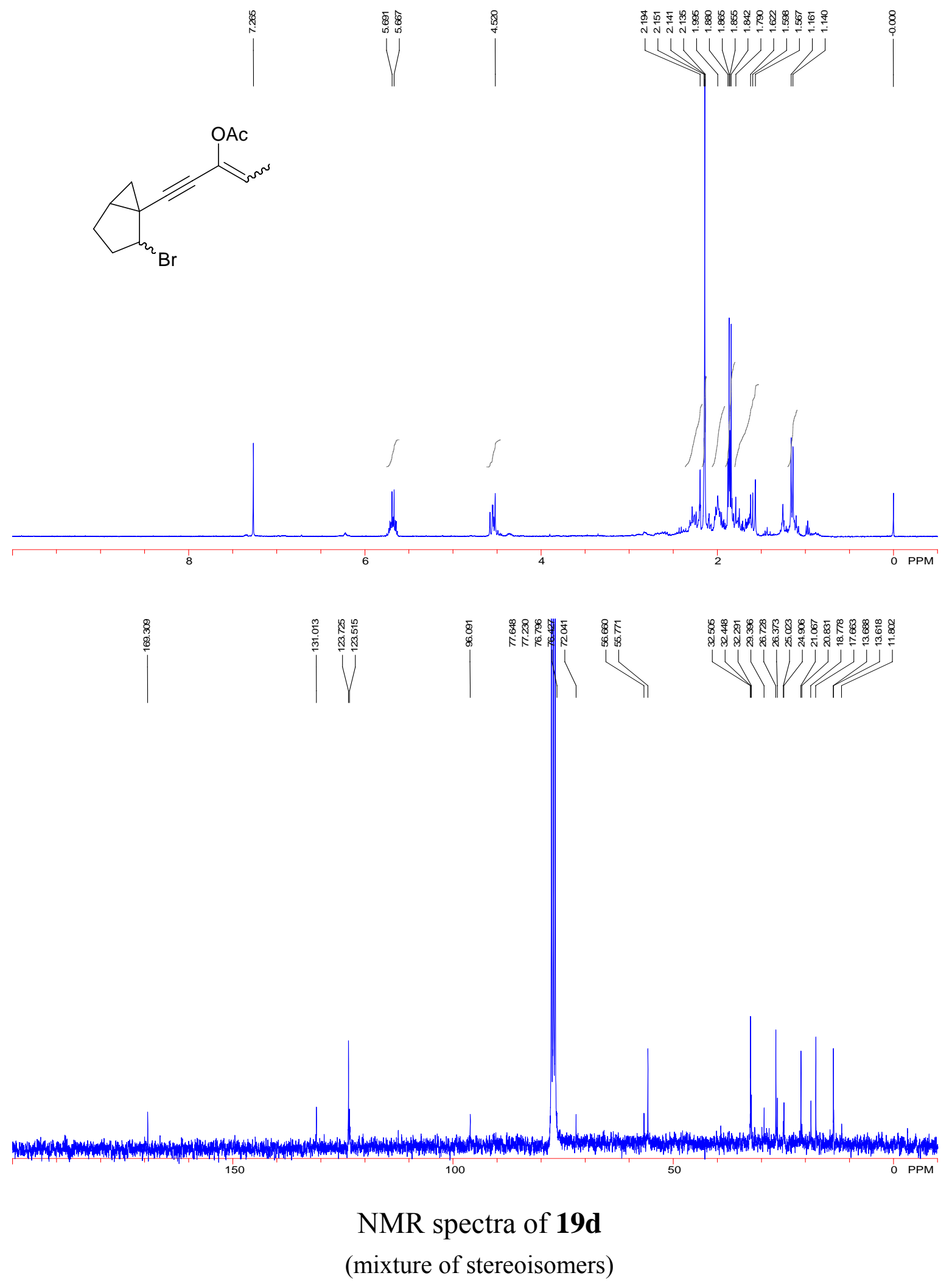

